\title{
Another View on the Middle East Conflict Analysis
}

\author{
Noor Rehman ${ }^{1,+} \oplus$, Syed Inayat Ali Shah ${ }^{2,+}{ }^{4}$, Abbas Ali ${ }^{1,+}{ }^{\text {, }}$, Sun Young Jang ${ }^{3,+, *}$ and \\ Choonkil Park $4,+, *$ (D) \\ 1 Department of Mathematics and Statistics, Riphah International University, I-14, Islamabad 44000, Pakistan; \\ noorrehman82@yahoo.com (N.R.); abbasali5068@gmail.com (A.A.) \\ 2 Department of Mathematics, Islamia College University, Peshawar 25120, KPK, Pakistan; \\ inayat64@gmail.com \\ 3 Department of Mathematics, University of Ulsan, Ulsan 44610, Korea \\ 4 Department of Mathematics, Research Institute of Natural Sciences, Hanyang University, Seoul 04763, Korea \\ * Correspondences: jsym@ulsan.ac.kr (S.Y.J.); baak@hanyang.ac.kr (C.P.); \\ Tel.: +82-10-9392-8439 (S.Y.J.); +82-2-2220-0892 (C.P.) \\ $\dagger$ These authors contributed equally to this work.
}

Received: 10 August 2018; Accepted: 31 August 2018; Published: 3 September 2018

\begin{abstract}
Decision making is a cognitive process for evaluating data with certain attributes to come up with the best option, in terms of the preferences of decision makers. Conflicts and disagreements occur in most real world problems and involve the applications of mathematical tools dealing with uncertainty, such as rough set theory in decision making and conflict analysis processes. Afterwards, the Pawlak conflict analysis model based on rough set theory was established. Subsequently, Deja put forward some questions that are not answered by the Pawlak conflict analysis model and Sun's model. In the present paper, using the notions of soft preference relation, soft dominance relation, and their roughness, we analyzed the Middle East conflict and answered the questions posed by Deja in a good manner.
\end{abstract}

Keywords: making decision; conflict analysis; rough set theory; soft preference relation; soft dominance relation

\section{Introduction}

In daily life, we always face various decision making pursuits. Some decision are simple and some are complex. When one faces a complex problem with conflicting criteria, an effective decision making method becomes helpful to make an acceptable and reasonable decision. Decision making is a key component for achieving objectives in diverse areas. Usually a conflict occurs when two people have different points of view about the same event or thing. The study of conflict is of utmost importance both practically and theoretically. No doubt its importance is increasing nowadays, as huge social networks based on cell phones, tablets, computers, and other gadgets and systems of computers play a paramount role in society [1]. There is always an uncertainty about agreement, disagreement, and neutrality in a conflict situation among agents. To deal with such situations, the main aim is how to model the uncertainty in conflict [2].

Pawlak applied rough set theory to the study of conflict analysis in Reference [3]. He also found a mathematical formulation for a conflict situation based on agreement, disagreement, and neutrality, and gave the axioms for these sets. Subsequently, Deja put forward an extension and generalization of Pawlak's model by adding some local aspects [2]. Pawlak conflict analysis model has certain limitations, for example, it cannot deal with intrinsic causes in the Middle East conflict. Another flaw of Pawlak's model is that, it has failed to find a feasible consensus strategy for solving a conflict. In Reference [4], Sun et al. developed a conflict analysis model based on rough set theory over two 
universes to overcome the above mentioned shortcomings in Pawlak's conflict analysis. But in their proposals, still there are many areas for critique, for example, the development of a proper and feasible consensus strategy is missing.

As a general mathematical tool while dealing with uncertainty, Molodtsov proposed soft set theory in Reference [5]. Maji et al. further strengthened the theory; they introduced several algebraic operations and examined their basic properties in Reference [6], which was later corrected by Ali et al. [7]. More on soft equivalence relations can be seen in Reference [8]. Applications of soft sets (hybrid soft sets) in decision making problems have been widely studied by many authors in different contexts (see References [9-24]). Many decision making problems have been characterized by the ranking of objects according to a set of criteria with predefined preference ordered decision classes, such as credit approval, stock risk estimation, university ranking, teaching evaluation, etc. (see References [25-27]). A recent work on the tolerance rough set can be found in Reference [28]. Many authors used the game theoretic rough sets model to construct a mechanism for analyzing the uncertainties of rough set theory [29-32]. In the present paper, we developed a new conflict analysis model, which is based on soft preference relation and soft dominance relation, to study the Middle East conflict situation. Further, we answered different questions raised by various authors in an affirmative way. A general technique for the conflict problem is proposed and examined and our newly developed model is more efficient than the existing techniques/models.

The rest of the paper is arranged as follows: Section 2 briefly reviews the Pawlak conflict analysis model followed by the Deja, together with Sun et al., conflict analysis models. Drawbacks of the Sun et al. conflict model are also discussed in this section. Section 3 consists of the proposed conflict analysis model based on soft set theory. Various uncertainties have been studied, which tried to address the shortcomings of Sun's model.

\section{Literature Review}

In the following, different conflict models are presented.

\subsection{Pawlak's Conflict Analysis Model}

The conflict analysis model introduced by Pawlak is based on rough set theory [3]. The Middle East conflict analysis problem is shown in Table 1, where the agents are represented by rows and the issues by columns. Each agent is assigned a value to an issue from the set $\{-, 0,+\}$, where - shows that the agent is against that particular issue, 0 shows the neutrality of the agent, and + depicts the favorability of the agent towards the issue/event. Furthermore, Pawlak discussed the Middle East conflict analysis problem in Reference [3]. This conflict analysis problem consists of five issues and six agents. The relationship of each agent to an issue is given in Table 1.

Let $U=\left\{u_{1}, u_{2}, u_{3}, u_{4}, u_{5}, u_{6}\right\}$ be the universe of six agents, where

$$
\begin{array}{lll}
u_{1}: \text { Israel } & u_{2}: \text { Egypt } & u_{3}: \text { Palestine } \\
u_{4}: \text { Jordan } & u_{5}: \text { Syria } & u_{6}: \text { Saudi Arabia. }
\end{array}
$$

Let $V=\left\{a_{1}, a_{2}, a_{3}, a_{4}, a_{5}\right\}$ be the universe of the five issues of the conflict situation, where

$a_{1}$ : Autonomous Palestinian state on the West Bank and Gaza,

$a_{2}$ : Israeli military outpost along the Jordan River,

$a_{3}$ : Israel retains East Jerusalem,

$a_{4}$ : Israeli military outposts on the Golan Heights, and

$a_{5}$ : Arab countries grant citizenship to Palestinians who choose to remain within their boarders. 
Table 1. Information system for the Middle East conflict.

\begin{tabular}{llllll}
\hline $\boldsymbol{U} \backslash \boldsymbol{V}$ & $\boldsymbol{a}_{\mathbf{1}}$ & $\boldsymbol{a}_{\mathbf{2}}$ & $\boldsymbol{a}_{\mathbf{3}}$ & $\boldsymbol{a}_{\mathbf{4}}$ & $\boldsymbol{a}_{\mathbf{5}}$ \\
\hline$u_{1}$ & - & + & + & + & + \\
$u_{2}$ & + & 0 & - & - & - \\
$u_{3}$ & + & - & - & - & 0 \\
$u_{4}$ & 0 & - & - & 0 & - \\
$u_{5}$ & + & - & - & - & - \\
$u_{6}$ & 0 & + & - & 0 & + \\
\hline
\end{tabular}

In the information system, the attitudes of the six nations of the Middle East region towards the above issues are presented.

\subsection{Deja's Conflict Analysis Model}

The conflict analysis of Reference [3] is confined to the conclusions, such as to find the most conflicted attribute or the assembly of agents when three or more take part in the conflict. Although Pawlak's conflict model has proven to be effective in practice, Deja in Reference [2], posed three questions that Pawlak's conflict model is unable to answer:

(i) What are the intrinsic reasons for the conflict?

(ii) How can a feasible consensus strategy be found?

(iii) Is it possible to satisfy all the agents?

\subsection{Sun et al.'s Conflict Analysis Model}

Sun et al. in Reference [4] tried to answer Deja's questions and focussed on answering the first and second questions raised by Deja in Reference [2].

Let $U=\left\{u_{1}, \ldots, u_{m}\right\}$ be the universe of agents and $V=\left\{a_{1}, \ldots, a_{n}\right\}$ be the universe of issues in the conflict situation. For any subset $A_{2}$ of $V$, we call $A_{2}$ the feasible strategy for a conflict situation. Let the set $f=\left\{f^{+}, f^{-}\right\}$be the collection of set valued mappings defined as follows:

$$
f^{+}: U \rightarrow P(V), f^{+}(u)=\{a \in V \mid f(u, a)=+\}, \text { for all } u \in U
$$

and:

$$
f^{-}: U \rightarrow P(V), f^{-}(u)=\{a \in V \mid f(u, a)=-\}, \text { for all } u \in U .
$$

The mapping $f^{+}$gives the subset of issues with which agent $u$ agrees while the mapping $f^{-}$gives the subset of issues with which the agent $u$ disagrees. For any feasible strategy $A_{2} \in \mathcal{P}(V)$ on an issue, the approximations are:

$$
\begin{gathered}
\underline{\operatorname{apr}}_{f}^{+}\left(A_{2}\right)=\left\{u \in U \mid f^{+}(u) \subseteq A_{2}\right\}, \underline{\operatorname{apr}}_{f}^{-}\left(A_{2}\right)=\left\{u \in U \mid f^{-}(u) \subseteq A_{2}\right\}, \\
\overline{a p r}_{f}^{+}\left(A_{2}\right)=\left\{u \in U \mid f^{+}(u) \cap A_{2} \neq \varnothing\right\}, \overline{a p r}_{f}^{-}\left(A_{2}\right)=\left\{u \in U \mid f^{-}(u) \cap A_{2} \neq \varnothing\right\} .
\end{gathered}
$$

Example 1. Consider the Middle East conflict as in Table 1.

Let $A_{2}=\left\{a_{2}, a_{3}, a_{5}\right\} \subseteq V$. Then $\underline{\text { apr }_{f}^{+}}\left(A_{2}\right)=\left\{u_{6}\right\}, \overline{a p r}_{f}^{+}\left(A_{2}\right)=\left\{u_{1}, u_{6}\right\}, \underline{\operatorname{apr}_{f}^{-}}\left(A_{2}\right)=\left\{u_{4}, u_{6}\right\}$, and $\overline{\operatorname{apr}}_{f}^{-}\left(A_{2}\right)=\left\{u_{2}, u_{3}, u_{4}, u_{5}, u_{6}\right\}$.

According to Sun et al. [4]:

Agreement subset $=R_{f}^{+}\left(A_{2}\right)=\underline{a p r}_{f}^{+}\left(A_{2}\right)-\underline{a p r}_{f}^{-}\left(A_{2}\right)=\varnothing$,

Disagreement subset $=R_{f}^{-}\left(A_{2}\right)=\underline{a_{f}}-\left(A_{2}\right)-\underline{a p r}_{f}^{+}\left(A_{2}\right)=\left\{u_{4}\right\}$,

Neutral subset $=U-\left(R_{f}^{+}\left(A_{2}\right) \cup R_{f}^{-}\left(A_{2}\right)\right)=\left\{u_{1}, u_{2}, u_{3}, u_{5}, u_{6}\right\}$. 
It is observed that:

(i) For the feasible strategy $A_{2}=\left\{a_{2}, a_{3}, a_{5}\right\} \in P(V)$ using Sun et al.'s technique, the agreement subset $=\varnothing$, the disagreement subset $=\left\{u_{4}\right\}$, and the neutral subset $=\left\{u_{1}, u_{2}, u_{3}, u_{5}, u_{6}\right\}$. This is because, in the information system (Table 1 ) the values for $u_{4}$ in the columns of $a_{2}, a_{3}$ and $a_{5}$ are in disagreement, that is " - ". Additionally, the case of $u_{5}$ is not different from $u_{4}$, but in Reference [4] it is treated as neutral, so the agents of the same behavior have been grouped in different coalitions, which causes confusion.

(ii) For the feasible strategy $A_{2}=\left\{a_{3}\right\} \in P(V)$, the agreement subset $=\varnothing$, the disagreement subset $=\left\{u_{6}\right\}$, and the neutral subset $=\left\{u_{1}, u_{2}, u_{3}, u_{4}, u_{5}\right\}$. This is because, in the information system (Table 1 ) the attitude of $u_{6}$ in the column of $a_{3}$ is in disagreement, that is "-". Also the cases of $u_{2}, u_{3}, u_{4}$ and $u_{5}$ are not different from $u_{6}$, but according to Reference [4], they should be in neutral, so the agents of the same character are grouped in different coalitions, which is an infirmity of the Sun et al. technique.

(iii) For another strategy, $A_{2}=\left\{a_{1}, a_{3}\right\} \in P(V)$, the agreement subset $=\left\{u_{2}, u_{3}, u_{5}\right\}$, the disagreement subset $=\left\{u_{1}, u_{6}\right\}$ and the neutral subset $=\left\{u_{4}\right\}$. Now, in the information system (Table 1) the behavior of $u_{4}$ and $u_{6}$ in the columns of $a_{1}$ and $a_{3}$ are the same. But using technique of Reference [4], these agents of the same character fall in different coalitions, which is another infirmity of the Sun et al. technique.

(iv) The technique of Reference [4] declared the conflict problem as an undecided one, which is another flaw of this technique as seen below:

Let $V=\left\{a_{1}, a_{2}, a_{3}, a_{4}, a_{5}\right\}$ be the set of all issues. Then

$\underline{\operatorname{apr}}_{f}^{+}(V)=\left\{u_{1}, u_{2}, u_{3}, u_{4}, u_{5}, u_{6}\right\}$ ，

$\operatorname{apr}_{f}^{-}(V)=\left\{u_{1}, u_{2}, u_{3}, u_{4}, u_{5}, u_{6}\right\}$,

the agreement subset $=\varnothing$, the disagreement subset $=\varnothing$, and the neutral subset $=$ $\left\{u_{1}, u_{2}, u_{3}, u_{4}, u_{5}, u_{6}\right\}$.

So for a feasible consensus strategy when $V=\left\{a_{1}, a_{2}, a_{3}, a_{4}, a_{5}\right\}$, the set of all issues of the conflict situation, there is no agreement subset and disagreement subset; the attitudes of all agents $u_{1}, u_{2}, u_{3}, u_{4}, u_{5}$, and $u_{6}$ are neutral for the feasible consensus strategy $V$.

\section{Conflict Analysis Based on Soft Preference Relation}

Inspired by the existing studies on conflict analysis based on Pawlak's rough set theory, as well as Sun et al.'s conflict analysis based on rough set theory over two universes, we propose a new conflict analysis model based on a soft preference relation that will help to improve the above limitations in the existing approaches in the literature.

In order to overcome the above mentioned shortcomings, a new conflict analysis model with the help of soft set theory is developed which is free from all such weaknesses and works more efficaciously.

Let $U$ be a set of agents and $V$ be a set of issues. Let $\succeq_{a}$ be an outranking relation on $U$ with respect to issue $a \in V$ such that $x_{1} \succeq_{a} x_{2}$ means " $x_{1}$ is at least as good as $x_{2}$ with respect to criterion $a$. Suppose that $\succeq_{a}$ is a complete preorder, it is strongly complete (which means that for each $x_{1}, x_{2} \in U$ at least one of $x_{1} \succeq_{a} x_{2}$ or $x_{2} \succeq_{a} x_{1}$ exists) and a transitive binary relation defined on $U$. Thus $x_{1}$ and $x_{2}$ are always comparable with respect to criterion $a \in V$. We say that an object $x_{1} E$-dominates an object $x_{2}$ with respect to $E \subseteq V$ (denoted by $x_{1} D_{E} x_{2}$ ), if $x_{1} \succeq_{a} x_{2}$ for all $a \in E$. Since the intersection of complete preorders is a partial preorder, $D_{E}=\bigcap_{a \in E} \succeq_{a}$, so the dominance relation $D_{E}$ is a partial preorder. 
Definition 1. [5] A pair $\Re=(F, E)$ is called a soft set over $U$, where $E$ is a subset of parameters of $V$ and $F: E \rightarrow \mathcal{P}(U)$ is a set valued mapping.

Definition 2. [8] If $F: E \rightarrow \mathcal{P}(U \times U)$ is a mapping from a subset of parameters $E$ to the power set of $U \times U$, then the soft set $(F, E)$ over $U \times U$ is called a soft binary relation over $U$. A soft binary relation $(F, E)$ over a set $U$ is called a soft equivalence relation over $U$, if $\varnothing \neq F(a)$ is an equivalence relation over $U$ for all $a \in E$.

Definition 3. Let $\mathcal{I S}=(U, V, \widetilde{f})$ be the Middle East conflict situation and $F: E \rightarrow \mathcal{P}(U \times U)$ be a set valued mapping from $E$ to $\mathcal{P}(U \times U)$, where $E \subseteq V$ denotes the set of parameters or attributes and $\mathcal{P}(U \times U)$ the set of all subsets of $U \times U$. If $\varnothing \neq F(a)$ is a preference relation for all $a \in E$, then $(F, E)$ is called a soft preference relation, where:

$$
F(a)=\left\{u_{i} \succeq u_{j} \mid \tilde{f}\left(u_{i}, a\right) \geq \tilde{f}\left(u_{j}, a\right), u_{i}, u_{j} \in U\right\}
$$

and:

$$
\tilde{f}: U \times V \rightarrow\{-, 0,+\},
$$

such that $\widetilde{f}(u, a)=s$, where $s \in\{-, 0,+\}$ and $u \in U, a \in V$. In fact $\widetilde{f}$ is more general than Sun et al.'s $f$ of Reference [4]. Throughout the paper, we will use $\left(u_{i}, u_{j}\right)$ for $u_{i} \succeq u_{j}$. Preference relations are efficient tools to address decision making problems that only require the decision makers to compare one pair of objects at a time. In the information system (Table 1), favorability $(+)$ is preferred to neutrality $(0)$, while neutrality $(0)$ is preferred to antagonism $(-)$.

Definition 4. Let $\mathcal{I} \mathcal{S}=(U, V, \widetilde{f})$ be the Middle East conflict situation and $(F, E)$ be a soft preference relation over $U$. Then there is an associated dominance relation, which can be denoted by $\mathcal{D} o \min \left(\mathcal{F}_{1}, E\right)$ and is defined as follows:

$$
\mathcal{D} o \min \left(\mathcal{F}_{1}, E\right)=\bigcap_{a \in E} F(a) .
$$

For any $u \in U$, define the soft dominance classes by:

$$
[u]_{\mathcal{D} o \min \left(\mathcal{F}_{1}, E\right)}^{+}=\left\{u_{i} \in U: u_{i} \succeq_{\mathcal{D} o \min \left(\mathcal{F}_{1}, E\right)} u\right\}
$$

and:

$$
[u]_{\mathcal{D} o \min \left(\mathcal{F}_{1}, E\right)}^{-}=\left\{u_{i} \in U: u_{i} \preceq_{\mathcal{D} o \min \left(\mathcal{F}_{1}, E\right)} u\right\},
$$

which represent the $(\mathcal{F}, E)$-dominating set and $(\mathcal{F}, E)$-dominated set with respect to $u \in U$ of the $\mathcal{I} \mathcal{S}$, respectively. Class $[u]_{\mathcal{D} o \min \left(\mathcal{F}_{1}, E\right)}^{+}$describes the set of all those objects that dominate $u$ and $[u]_{\mathcal{D} o \min \left(\mathcal{F}_{1}, E\right)}^{-}$ describes the set of all those objects that are dominated by $u$ in terms of $\mathcal{D}$ o $\min \left(\mathcal{F}_{1}, E\right)$, where:

$$
\mathcal{D} o \min \left(\mathcal{F}_{1}, E\right)^{+}=\left\{u \in U:[u]_{\mathcal{D} o \min \left(\mathcal{F}_{1}, E\right)}^{+}\right\}
$$

and:

$$
\mathcal{D} o \min \left(\mathcal{F}_{1}, E\right)^{-}=\left\{u \in U:[u]_{\mathcal{D} o \min \left(\mathcal{F}_{1}, E\right)}^{-}\right\} \text {. }
$$

Theorem 1. Let $\mathcal{I} \mathcal{S}=(U, V, \widetilde{f})$ be the Middle East conflict situation and $\mathcal{D} o \min \left(\mathcal{F}_{1}, E\right)$ be a soft dominance relation. Then the following hold:

(1) If $E_{1} \subseteq E$, then $\mathcal{D} o \min \left(\mathcal{F}_{1}, E\right) \subseteq \mathcal{D} o \min \left(\mathcal{F}_{1}, E_{1}\right)$;

(2) If $a_{n} \in\left[a_{m}\right]_{\mathcal{D} o \min \left(\mathcal{F}_{1}, E\right)}^{+}$, then $\left[a_{n}\right]_{\mathcal{D} o \min \left(\mathcal{F}_{1}, E\right)}^{+} \subseteq\left[a_{m}\right]_{\mathcal{D} o \min \left(\mathcal{F}_{1}, E\right)}^{+}$;

(3) $\left[a_{m}\right]_{\mathcal{D} o \min \left(\mathcal{F}_{1}, E\right)}^{+}=\bigcup\left\{\left[a_{n}\right]_{\mathcal{D} o \min \left(\mathcal{F}_{1}, E\right)}^{-}: a_{n} \in\left[a_{m}\right]_{\mathcal{D} o \min \left(\mathcal{F}_{1}, E\right)}^{+}\right\}$; 
(4) $\left[a_{m}\right]_{\mathcal{D o m}^{k}(\mathcal{H}, \mathrm{C})}^{+}=\left[a_{n}\right]_{{\mathcal{D} o m^{k}}^{(\mathcal{H}, C)}}^{+}$iff $f\left(a_{m}, C_{j}\right)=f\left(a_{n}, C_{j}\right)$ and $g\left(a_{m}, D_{k}\right)=g\left(a_{n}, D_{k}\right)$ for all $C_{j} \in C$ and $D_{k} \in D$;

(5) $\Gamma=\left\{\left[a_{m}\right]_{\mathcal{D} o \min \left(\mathcal{F}_{1}, E\right)}^{+}: a_{m} \in U\right\}$ constitutes a covering of $U$.

Proof. The proof is straightforward.

Definition 5. Let $\mathcal{I} \mathcal{S}=(U, V, \widetilde{f})$ be the Middle East conflict situation. Then, for any $A_{1} \subseteq U$ the lower and upper approximations with respect to the $\left(\mathcal{F}_{1}, E\right)$-dominating set and $\left(\mathcal{F}_{1}, E\right)$-dominated set are:

$$
\begin{gathered}
\left(\underline{A_{1}}\right)_{\mathcal{D} o \min \left(\mathcal{F}_{1}, E\right)^{+}}=\left\{u \in U:[u]_{\mathcal{D} o \min \left(\mathcal{F}_{1}, E\right)}^{+} \subseteq A_{1}\right\} \\
\left(\overline{A_{1}}\right)_{\mathcal{D} o \min \left(\mathcal{F}_{1}, E\right)^{+}}=\left\{u \in U:[u]_{\mathcal{D} o \min \left(\mathcal{F}_{1}, E\right)}^{-} \cap A_{1}: \neq \varnothing\right\} .
\end{gathered}
$$

The lower approximation $\left(\underline{A_{1}}\right)_{\mathcal{D} o \min \left(\mathcal{F}_{1}, E\right)^{+}}$, is the collection of all those objects $u$ from $U$, such that all objects $u_{i}$, having at least the same evaluations on all of the considered criteria from $V$, also belong to $A_{1}$. Thus one can say that if an object $u_{i}$ has at least as good an evaluation on the criteria from $V$ as an object $u$ belonging to $\left(\underline{A_{1}}\right)_{\mathcal{D} o \min \left(\mathcal{F}_{1}, E\right)^{+}}$, then certainly $u_{i}$ belongs to $A_{1}$. The upper approximation $\left(\overline{A_{1}}\right)_{\mathcal{D} o \min \left(\mathcal{F}_{1}, E\right)^{+}}$ is the collection of all those objects $u$ from $U$ whose evaluation on the criteria from $V$ is not worse than the

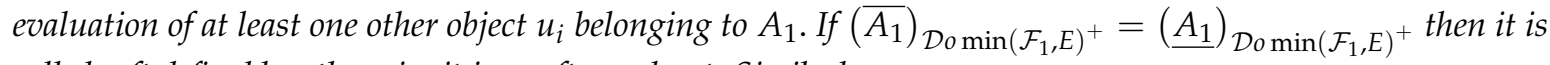
called soft definable, otherwise it is a soft rough set. Similarly:

$$
\begin{gathered}
\left(\underline{A_{1}}\right)_{\mathcal{D} o \min \left(\mathcal{F}_{1}, E\right)}=\left\{u \in U:[u]_{\mathcal{D} o \min \left(\mathcal{F}_{1}, E\right)}^{-} \subseteq A_{1}\right\} \\
\left(\overline{A_{1}}\right)_{\mathcal{D}_{o \min \left(\mathcal{F}_{1}, E\right)^{-}}}=\left\{u \in U:[u]_{\mathcal{D} o \min \left(\mathcal{F}_{1}, E\right)}^{+} \cap A_{1}: \neq \varnothing\right\} .
\end{gathered}
$$

If $\left(\overline{A_{1}}\right)_{\mathcal{D} o \min \left(\mathcal{F}_{1}, E\right)^{-}}=\left(\underline{A_{1}}\right)_{\mathcal{D} o \min \left(\mathcal{F}_{1}, E\right)^{-}}$then it is called soft definable, otherwise it is a soft rough set. We call,

(1) $\operatorname{POS}\left(\left(A_{1}\right)_{\mathcal{D o} \min \left(\mathcal{F}_{1}, E\right)^{+}}\right)=\left(\underline{A_{1}}\right)_{\mathcal{D} \text { o } \min \left(\mathcal{F}_{1}, E\right)^{+}}$the positive region of $A_{1}$ with respect to $\mathcal{D} o \min \left(\mathcal{F}_{1}, E\right)^{+}$.

(2) $\quad N E G\left(\left(A_{1}\right)_{\mathcal{D} o \min \left(\mathcal{F}_{1}, E\right)^{+}}\right)=U-\left(\overline{A_{1}}\right)_{\mathcal{D} o \min \left(\mathcal{F}_{1}, E\right)^{+}}$the negative region of $A_{1}$ with respect to $\mathcal{D} o \min \left(\mathcal{F}_{1}, E\right)^{+}$.

(3) $\quad B N\left(\left(A_{1}\right)_{\mathcal{D} o \min \left(\mathcal{F}_{1}, E\right)^{+}}\right)=\left(\overline{A_{1}}\right)_{\mathcal{D} o \min \left(\mathcal{F}_{1}, E\right)^{+}}-\left(\underline{A_{1}}\right)_{\mathcal{D} o \min \left(\mathcal{F}_{1}, E\right)^{+}}$the boundary region of $A_{1}$ with respect to $\mathcal{D} o \min \left(\mathcal{F}_{1}, E\right)^{+}$in information system $\mathcal{I S}$.

(4) $\operatorname{POS}\left(\left(A_{1}\right)_{\mathcal{D} o \min \left(\mathcal{F}_{1}, E\right)^{-}}\right)=\left(\underline{A_{1}}\right)_{\mathcal{D} o \min \left(\mathcal{F}_{1}, E\right)^{-}}$the positive region of $A_{1}$ with respect to $\operatorname{Do} \min \left(\mathcal{F}_{1}, E\right)^{-}$

(5) $\quad \operatorname{NEG}\left(\left(A_{1}\right)_{\mathcal{D} o \min \left(\mathcal{F}_{1}, E\right)^{-}}\right)=U-\left(\overline{A_{1}}\right)_{\mathcal{D} o \min \left(\mathcal{F}_{1}, E\right)^{-}}$the negative region of $A_{1}$ with respect to $\mathcal{D} o \min \left(\mathcal{F}_{1}, E\right)^{-}$.

(6) $\quad B N\left(\left(A_{1}\right)_{\mathcal{D} o \min \left(\mathcal{F}_{1}, E\right)^{-}}\right)=\left(\overline{A_{1}}\right)_{\mathcal{D} \text { o } \min \left(\mathcal{F}_{1}, E\right)^{-}}-\left(\underline{A_{1}}\right)_{\mathcal{D} \text { o } \min \left(\mathcal{F}_{1}, E\right)^{-}}$the boundary region of $A_{1}$ with respect to $\mathcal{D}$ o $\min \left(\mathcal{F}_{1}, E\right)^{-}$in information system $\mathcal{I} \mathcal{S}$.

Theorem 2. Let $\mathcal{I} \mathcal{S}=(U, V, \widetilde{f})$ be the Middle East conflict situation. Then for any $A_{1}, A_{2} \subseteq U$ :

(1) $\left(\underline{A}_{1}\right)_{\mathcal{D} o \min \left(\mathcal{F}_{1}, E\right)^{+}} \subseteq A_{1} \subseteq\left(\overline{A_{1}}\right)_{\mathcal{D} o \min \left(\mathcal{F}_{1}, E\right)^{+}}$;

(2) $(\underline{\varnothing})_{\mathcal{D o m i n}\left(\mathcal{F}_{1}, E\right)^{+}}=(\bar{\varnothing})_{\mathcal{D}_{o} \min \left(\mathcal{F}_{1}, E\right)^{+}}=\varnothing$;

(3) $(\underline{U})_{\mathcal{D o m i n}\left(\mathcal{F}_{1}, E\right)^{+}}:=(\bar{U})_{\mathcal{D}_{0 \text { min }\left(\mathcal{F}_{1}, E\right)^{+}}=U \text {; }}$

(4) $A_{1} \subseteq A_{2}$ implies $\left(\underline{A_{1}}\right)_{\mathcal{D o} \min \left(\mathcal{F}_{1}, E\right)^{+}} \subseteq\left(\underline{A_{2}}\right)_{\mathcal{D o m i n}\left(\mathcal{F}_{1}, E\right)^{+}}$; 
(5) $A_{1} \subseteq A_{2}$ implies $\left(\overline{A_{1}}\right)_{\mathcal{D o m i n}\left(\mathcal{F}_{1}, E\right)^{+}} \subseteq\left(\overline{A_{2}}\right)_{\mathcal{D} o \min \left(\mathcal{F}_{1}, E\right)^{+}}$;

(6) $\left(\underline{A}_{1}\right)_{\mathcal{D} o \min \left(\mathcal{F}_{1}, E\right)^{+}} \cup\left(\underline{A_{2}}\right)_{\mathcal{D} o \min \left(\mathcal{F}_{1}, E\right)^{+}} \subseteq\left(\underline{\left(A_{1}\right.}\right)_{\mathcal{D} o \min \left(\mathcal{F}_{1}, E\right)^{+}}$;

(7) $\left(\overline{A_{1}}\right)_{\mathcal{D} o \min \left(\mathcal{F}_{1}, E\right)^{+}} \cup\left(\overline{A_{2}}\right)_{\mathcal{D} o \min \left(\mathcal{F}_{1}, E\right)^{+}}=\left(\overline{A_{1} \cup A_{2}}\right)_{\mathcal{D} o \min \left(\mathcal{F}_{1}, E\right)^{+}}$;

(8) $\quad\left(\underline{A_{1}}\right)_{\mathcal{D} o \min \left(\mathcal{F}_{1}, E\right)^{+}} \cap\left(\underline{A_{2}}\right)_{\mathcal{D} o \min \left(\mathcal{F}_{1}, E\right)^{+}}=\left(\underline{A}_{1}\right)_{\mathcal{D o m i n}\left(\mathcal{F}_{1}, E\right)^{+}}$;

(9) $\left(\overline{A_{1}}\right)_{\mathcal{D} o \min \left(\mathcal{F}_{1}, E\right)^{+}} \cap\left(\overline{A_{2}}\right)_{\mathcal{D} o \min \left(\mathcal{F}_{1}, E\right)^{+}} \supseteq\left(\overline{A_{1} \cap A_{2}}\right)_{\mathcal{D o m i n}\left(\mathcal{F}_{1}, E\right)^{+}}$;

Proof. These results follow from the respective definitions.

The following theorems give a connection between topology and soft dominating/dominated definable sets.

Theorem 3. Let $\mathcal{I} \mathcal{S}=(U, V, \widetilde{f})$ be the Middle East conflict situation. Then the collection of soft dominating definable sets form a topology on $U$.

Proof. (i) From Theorem 2:

$$
(\underline{\varnothing})_{\mathcal{D} o \min \left(\mathcal{F}_{1}, E\right)^{+}}=(\bar{\varnothing})_{\mathcal{D} o \min \left(\mathcal{F}_{1}, E\right)^{+}}=\varnothing
$$

and:

$$
(\underline{U})_{\mathcal{D} o \min \left(\mathcal{F}_{1}, E\right)^{+}}=(\bar{U})_{\mathcal{D} o \min \left(\mathcal{F}_{1}, E\right)^{+}}=U .
$$

(ii) We prove that an arbitrary union of soft dominating definable sets is a soft dominating definable set. For this consider:

$$
\begin{aligned}
\left(\overline{\bigcup_{i} A_{i}}\right)_{\mathcal{D} o \min \left(\mathcal{F}_{1}, E\right)^{+}} & =\underset{i}{\cup}\left(\overline{A_{i}}\right)_{\mathcal{D} o \min \left(\mathcal{F}_{1}, E\right)^{+}}=\bigcup_{i} \underline{\left(A_{i}\right)_{\mathcal{D} o \min \left(\mathcal{F}_{1}, E\right)^{+}}} \\
& \subseteq\left(\underline{\cup_{i} A_{i}}\right)_{\mathcal{D o m i n}\left(\mathcal{F}_{1}, E\right)^{+}}
\end{aligned}
$$

Similarly, $\left(\underline{\cup}_{\underline{i} A_{i}}\right)_{\mathcal{D} o \min \left(\mathcal{F}_{1}, E\right)^{+}} \subseteq\left(\overline{\bigcup_{i} A_{i}}\right)_{\mathcal{D} o \min \left(\mathcal{F}_{1}, E\right)^{+}}$.

Hence, $\left(\underline{\cup}_{\underline{i} A_{i}}\right)_{\mathcal{D o m i n}\left(\mathcal{F}_{1}, E\right)^{+}}=\left(\overline{\bigcup_{i} A_{i}}\right)_{\mathcal{D} o \min \left(\mathcal{F}_{1}, E\right)^{+}}$.

(iii) Last, we prove a finite intersection of soft dominating definable sets is a soft dominating definable set. For this consider:

$$
\begin{aligned}
\left(\overline{\prod_{i}^{n} A_{i}}\right)_{\mathcal{D} o \min \left(\mathcal{F}_{1}, E\right)^{+}} & \subseteq \Gamma_{i}^{n}\left(\overline{A_{i}}\right)_{\mathcal{D} o \min \left(\mathcal{F}_{1}, E\right)^{+}}=\bigcap_{i}^{n}\left(\underline{A_{i}}\right)_{\mathcal{D} o \min \left(\mathcal{F}_{1}, E\right)^{+}} \\
& =\left(\underline{\Gamma_{i}^{n} A_{i}}\right)_{\mathcal{D} o \min \left(\mathcal{F}_{1}, E\right)^{+}} .
\end{aligned}
$$

Similarly, $\left(\underline{\bigcap_{i}^{n} A_{i}}\right)_{\mathcal{D} o \min \left(\mathcal{F}_{1}, E\right)^{+}} \subseteq\left(\overline{\cap_{i}^{n} A_{i}}\right)_{\mathcal{D} o \min \left(\mathcal{F}_{1}, E\right)^{+}}$. Therefore, $\left({\underline{\left(\bigcap_{i}^{n} A_{i}\right.}}_{\mathcal{D}_{\operatorname{Domin}\left(\mathcal{F}_{1}, E\right)^{+}}}=\right.$ $\left(\overline{\bar{\cap}_{i}^{n} A_{i}}\right)_{\mathcal{D o m i n}\left(\mathcal{F}_{1}, E\right)^{+}}$.

Theorem 4. Let $\mathcal{I} \mathcal{S}=(U, V, \widetilde{f})$ be the Middle East conflict situation. Then the collection of soft dominated definable sets form a topology on $U$. 
Proof. The proof is similar to the proof of Theorem 3.

Definition 6. Let $\mathcal{I} \mathcal{S}=(U, V, \widetilde{f})$ be the Middle East conflict situation. For any $A_{1} \subseteq U$, the approximate precisions $\rho_{\mathcal{D} o \min \left(\mathcal{F}_{1}, E\right)^{+}}\left(A_{1}\right)$ and $\rho_{\mathcal{D} o \min \left(\mathcal{F}_{1}, E\right)^{-}}\left(A_{1}\right)$ of $A_{1}$ about $\mathcal{D}$ o $\min \left(\mathcal{F}_{1}, E\right)^{+}$and $\mathcal{D} o \min \left(\mathcal{F}_{1}, E\right)^{-}$ are defined as:

$$
\rho_{\mathcal{D} o \min \left(\mathcal{F}_{1}, E\right)^{+}}\left(A_{1}\right)=\frac{\left.\mid \underline{\left(A_{1}\right.}\right)_{\mathcal{D} o \min \left(\mathcal{F}_{1}, E\right)^{+}} \mid}{\left|\left(\overline{A_{1}}\right)_{\mathcal{D} o \min \left(\mathcal{F}_{1}, E\right)^{+}}\right|} \text {and } \rho_{\mathcal{D} o \min \left(\mathcal{F}_{1}, E\right)^{-}}\left(A_{1}\right)=\frac{\left.\mid \underline{\left(A_{1}\right.}\right)_{\mathcal{D} o \min \left(\mathcal{F}_{1}, E\right)^{-}} \mid}{\left|\left(\overline{A_{1}}\right)_{\mathcal{D} o \min \left(\mathcal{F}_{1}, E\right)^{-}}\right|},
$$

where $A_{1}: \neq \varnothing$, and $|\cdot|$ denotes the cardinality of a set.

$$
\text { Let } \mu_{\mathcal{D} o \min \left(\mathcal{F}_{1}, E\right)^{+}}\left(A_{1}\right)=1-\rho_{\mathcal{D} o \min \left(\mathcal{F}_{1}, E\right)^{+}}\left(A_{1}\right) \text { and } \mu_{\mathcal{D} o \min \left(\mathcal{F}_{1}, E\right)^{-}}\left(A_{1}\right)=1-\rho_{\mathcal{D} o \min \left(\mathcal{F}_{1}, E\right)^{-}}\left(A_{1}\right) \text {. }
$$

Then $\mu_{\mathcal{D} o \min \left(\mathcal{F}_{1}, E\right)^{+}}\left(A_{1}\right)$ and $\mu_{\mathcal{D} o \min \left(\mathcal{F}_{1}, E\right)^{-}}\left(A_{1}\right)$ are called the rough degrees of $A_{1}$ about $\mathcal{D}$ o $\min \left(\mathcal{F}_{1}, E\right)^{+}$ and $\mathcal{D} o \min \left(\mathcal{F}_{1}, E\right)^{-}$, respectively.

By definition $0 \leq \mu_{\mathcal{D o m i n}\left(\mathcal{F}_{1}, E\right)^{+}}\left(A_{1}\right) \leq 1$ and $0 \leq \rho_{\mathcal{D o m i n}\left(\mathcal{F}_{1}, E\right)^{+}}\left(A_{1}\right) \leq 1$. It can be seen that $\rho_{\mathcal{D o m i n}\left(\mathcal{F}_{1}, E\right)^{+}}\left(A_{1}\right)=1$ if and only if $\left(\underline{A_{1}}\right)_{\mathcal{D} o \min \left(\mathcal{F}_{1}, E\right)^{+}}=A_{1}=\left(\overline{A_{1}}\right)_{\mathcal{D} \text { o } \min \left(\mathcal{F}_{1}, E\right)^{+}}$. Similarly, $0 \leq \mu_{\mathcal{D o m i n}\left(\mathcal{F}_{1}, E\right)^{-}}\left(A_{1}\right) \leq 1$ and $0 \leq \rho_{\mathcal{D} o \min \left(\mathcal{F}_{1}, E\right)^{-}}\left(A_{1}\right) \leq 1$.

The following theorems describe the relationships of the precisions, $\rho_{\mathcal{D o} \min \left(\mathcal{F}_{1}, E\right)^{+}}\left(A_{1}\right)$ and $\rho_{\mathcal{D} o \min \left(\mathcal{F}_{1}, E\right)^{-}}\left(A_{1}\right)$, and rough degrees, $\mu_{\mathcal{D o m i n}\left(\mathcal{F}_{1}, E\right)^{+}}\left(A_{1}\right)$ and $\mu_{\mathcal{D o m i n}\left(\mathcal{F}_{1}, E\right)^{-}}\left(A_{1}\right)$, with the intersection and union of any subsets $A_{1}$ and $A_{2}$ of the universe $U$.

Theorem 5. Let $\mathcal{I} \mathcal{S}=(U, V, \widetilde{f})$ be the Middle East conflict situation and $A_{1}, A_{2} \subseteq U$. Then the rough degree and precision of the sets $A_{1}, A_{2}, A_{1} \cup A_{2}$, and $A_{1} \cap A_{2}$ satisfy the following relations:

(1)

$$
\begin{aligned}
& \mu_{\mathcal{D} o \min \left(\mathcal{F}_{1}, E\right)^{+}}\left(A_{1} \cup A_{2}\right)\left|\left(\overline{A_{1}}\right)_{\mathcal{D} o \min \left(\mathcal{F}_{1}, E\right)^{+}} \cup\left(\overline{A_{2}}\right)_{\mathcal{D} o \min \left(\mathcal{F}_{1}, E\right)^{+}}\right| \\
\leq & \mu_{\mathcal{D} o \min \left(\mathcal{F}_{1}, E\right)^{+}}\left(A_{1}\right)\left|\left(\overline{A_{1}}\right)_{\mathcal{D} o \min \left(\mathcal{F}_{1}, E\right)^{+}}\right|+\mu_{\mathcal{D} o \min \left(\mathcal{F}_{1}, E\right)^{+}}\left(A_{2}\right)\left|\left(\overline{A_{2}}\right)_{\mathcal{D} o \min \left(\mathcal{F}_{1}, E\right)^{+}}\right| \\
& -\mu_{\mathcal{D} o \min \left(\mathcal{F}_{1}, E\right)^{+}}\left(A_{1} \cap A_{2}\right)\left|\left(\overline{A_{1}}\right)_{\mathcal{D} o \min \left(\mathcal{F}_{1}, E\right)^{+}} \cap\left(\overline{A_{2}}\right)_{\mathcal{D} o \min \left(\mathcal{F}_{1}, E\right)^{+}}\right| .
\end{aligned}
$$

(2)

$$
\begin{aligned}
& \rho_{\mathcal{D} o \min \left(\mathcal{F}_{1}, E\right)^{+}}\left(A_{1} \cup A_{2}\right)\left|\left(\overline{A_{1}}\right)_{\mathcal{D} o \min \left(\mathcal{F}_{1}, E\right)^{+}} \cup\left(\overline{A_{2}}\right)_{\mathcal{D} o \min \left(\mathcal{F}_{1}, E\right)^{+}}\right| \\
\geq & \rho_{\mathcal{D} o \min \left(\mathcal{F}_{1}, E\right)^{+}}\left(A_{1}\right)\left|\left(\overline{A_{1}}\right)_{\mathcal{D} o \min \left(\mathcal{F}_{1}, E\right)^{+}}\right|+\rho_{\mathcal{D} o \min \left(\mathcal{F}_{1}, E\right)^{+}}\left(A_{2}\right)\left|\left(\overline{A_{2}}\right)_{\mathcal{D} o \min \left(\mathcal{F}_{1}, E\right)^{+}}\right| \\
& -\rho_{\mathcal{D} o \min \left(\mathcal{F}_{1}, E\right)^{+}}\left(A_{1} \cap A_{2}\right)\left|\left(\overline{A_{1}}\right)_{\mathcal{D} o \min \left(\mathcal{F}_{1}, E\right)^{+}} \cap\left(\overline{A_{2}}\right)_{\mathcal{D} o \min \left(\mathcal{F}_{1}, E\right)^{+}}\right|
\end{aligned}
$$

Proof. (1) By definition of the rough degree, we have:

$$
\begin{aligned}
\mu_{\mathcal{D} o \min \left(\mathcal{F}_{1}, E\right)^{+}}\left(A_{1} \cup A_{2}\right) & =1-\frac{\left|\left(\underline{A_{1}}\right)_{\mathcal{D} o \min \left(\mathcal{F}_{1}, E\right)^{+}}\right|}{\mid \overline{\left(\overline{A_{1} \cup A_{2}}\right)_{\mathcal{D} o \min \left(\mathcal{F}_{1}, E\right)^{+}}}} \\
& =1-\frac{\left|\left(\underline{A_{1}}\right)_{\mathcal{D} o \min \left(\mathcal{F}_{1}, E\right)^{+}}\right|}{\left|\left(\overline{A_{1}}\right)_{\mathcal{D} o \min \left(\mathcal{F}_{1}, E\right)^{+}} \cup\left(\overline{A_{2}}\right)_{\mathcal{D} o \min \left(\mathcal{F}_{1}, E\right)^{+}}\right|} \\
& \leq 1-\frac{\left|\left(\underline{A_{1}}\right)_{\mathcal{D} o \min \left(\mathcal{F}_{1}, E\right)^{+}} \cup\left(\underline{A_{2}}\right)_{\mathcal{D} o \min \left(\mathcal{F}_{1}, E\right)^{+}}\right|}{\left|\left(\overline{A_{1}}\right)_{\mathcal{D} o \min \left(\mathcal{F}_{1}, E\right)^{+}} \cup\left(\overline{A_{2}}\right)_{\mathcal{D} o \min \left(\mathcal{F}_{1}, E\right)^{+}}\right|} .
\end{aligned}
$$


Thus we obtain:

$$
\begin{aligned}
& \mu_{\mathcal{D} o \min \left(\mathcal{F}_{1}, E\right)^{+}}\left(A_{1} \cup A_{2}\right)\left|\left(\overline{A_{1}}\right)_{\mathcal{D} o \min \left(\mathcal{F}_{1}, E\right)^{+}} \cup\left(\overline{A_{2}}\right)_{\mathcal{D} o \min \left(\mathcal{F}_{1}, E\right)^{+}}\right| \\
\leq & \left|\left(\overline{A_{1}}\right)_{\mathcal{D} o \min \left(\mathcal{F}_{1}, E\right)^{+}} \cup\left(\overline{A_{2}}\right)_{\mathcal{D} o \min \left(\mathcal{F}_{1}, E\right)^{+}}\right|-\mid{\underline{\left(A_{1}\right.}}_{\mathcal{D}_{o \min \left(\mathcal{F}_{1}, E\right)^{+}} \cup\left(\underline{A_{2}}\right)_{\mathcal{D} o \min \left(\mathcal{F}_{1}, E\right)^{+}} \mid .}
\end{aligned}
$$

Similarly:

$$
\begin{aligned}
\mu_{\mathcal{D} o \min \left(\mathcal{F}_{1}, E\right)^{+}}\left(A_{1} \cap A_{2}\right) & =1-\frac{\left|\left(\underline{A_{1}}\right)_{\mathcal{D} o \min \left(\mathcal{F}_{1}, E\right)^{+}}\right|}{\left|\left(\overline{A_{1} \cap A_{2}}\right)_{\mathcal{D} o \min \left(\mathcal{F}_{1}, E\right)^{+}}\right|} \\
& =1-\frac{\mid \underline{\left(\underline{A_{1}}\right)_{\mathcal{D} o \min \left(\mathcal{F}_{1}, E\right)^{+}} \cap\left(\underline{A_{2}}\right)_{\mathcal{D} o \min \left(\mathcal{F}_{1}, E\right)^{+}} \mid}}{\left|\left(\overline{A_{1} \cap A_{2}}\right)_{\mathcal{D} o \min \left(\mathcal{F}_{1}, E\right)^{+}}\right|} \\
& \leq 1-\frac{\left.\mid \underline{\left(\bar{A}_{1}\right.}\right)_{\mathcal{D} o \min \left(\mathcal{F}_{1}, E\right)^{+}} \cap\left(\underline{A_{2}}\right)_{\mathcal{D} o \min \left(\mathcal{F}_{1}, E\right)^{+}} \mid}{\left|\left(\overline{A_{1}}\right)_{\mathcal{D} o \min \left(\mathcal{F}_{1}, E\right)^{+}} \cap\left(\overline{A_{2}}\right)_{\mathcal{D} o \min \left(\mathcal{F}_{1}, E\right)^{+}}\right|} .
\end{aligned}
$$

Hence:

$$
\begin{aligned}
& \mu_{\mathcal{D o m i n}\left(\mathcal{F}_{1}, E\right)^{+}}\left(A_{1} \cap A_{2}\right)\left|\left(\overline{A_{1}}\right)_{\mathcal{D} o \min \left(\mathcal{F}_{1}, E\right)^{+}} \cap\left(\overline{A_{2}}\right)_{\mathcal{D} o \min \left(\mathcal{F}_{1}, E\right)^{+}}\right| \\
& \leq\left|\left(\overline{A_{1}}\right)_{\mathcal{D} o \min \left(\mathcal{F}_{1}, E\right)^{+}} \cap\left(\overline{A_{2}}\right)_{\mathcal{D} o \min \left(\mathcal{F}_{1}, E\right)^{+}}\right|-\left|\left(\underline{A}_{1}\right)_{\mathcal{D} o \min \left(\mathcal{F}_{1}, E\right)^{+}} \cap\left(\underline{A}_{2}\right)_{\mathcal{D} o \min \left(\mathcal{F}_{1}, E\right)^{+}}\right| .
\end{aligned}
$$

We know that for any sets $|A \cup B|=|A|+|B|-|A \cap B|$. It follows that:

$$
\begin{aligned}
& \mu_{\mathcal{D} o \min \left(\mathcal{F}_{1}, E\right)^{+}}\left(A_{1} \cup A_{2}\right)\left|\left(\overline{A_{1}}\right)_{\mathcal{D} o \min \left(\mathcal{F}_{1}, E\right)^{+}} \cup\left(\overline{A_{2}}\right)_{\mathcal{D} o \min \left(\mathcal{F}_{1}, E\right)^{+}}\right| \\
& \leq\left|\left(\overline{A_{1}}\right)_{\mathcal{D} o \min \left(\mathcal{F}_{1}, E\right)^{+}} \cup\left(\overline{A_{2}}\right)_{\mathcal{D} o \min \left(\mathcal{F}_{1}, E\right)^{+}}\right|-\left|\left(\underline{A_{1}}\right)_{\mathcal{D} o \min \left(\mathcal{F}_{1}, E\right)^{+}} \cup\left(\underline{A_{2}}\right)_{\mathcal{D} o \min \left(\mathcal{F}_{1}, E\right)^{+}}\right| \\
& =\left|\left(\overline{A_{1}}\right)_{\mathcal{D} o \min \left(\mathcal{F}_{1}, E\right)^{+}}\right|+\left|\left(\overline{A_{2}}\right)_{\mathcal{D} o \min \left(\mathcal{F}_{1}, E\right)^{+}}\right|-\left|\left(\overline{A_{1}}\right)_{\mathcal{D} o \min \left(\mathcal{F}_{1}, E\right)^{+}} \cap\left(\overline{A_{2}}\right)_{\mathcal{D} o \min \left(\mathcal{F}_{1}, E\right)^{+}}\right| \\
& -\left|\left(\underline{A}_{1}\right)_{\mathcal{D} o \min \left(\mathcal{F}_{1}, E\right)^{+}}\right|-\left|\left(\underline{A_{2}}\right)_{\mathcal{D} o \min \left(\mathcal{F}_{1}, E\right)^{+}}\right|+\left|\left(\underline{\left(A_{1}\right.}\right)_{\mathcal{D} o \min \left(\mathcal{F}_{1}, E\right)^{+}} \cap\left(\underline{A_{2}}\right)_{\mathcal{D} o \min \left(\mathcal{F}_{1}, E\right)^{+}}\right| \\
& \left.=\left|\left(\overline{A_{1}}\right)_{\mathcal{D} o \min \left(\mathcal{F}_{1}, E\right)^{+}}\right|+\left|\left(\overline{A_{2}}\right)_{\mathcal{D} o \min \left(\mathcal{F}_{1}, E\right)^{+}}\right|-\mid \underline{\left(A_{1}\right.}\right)_{\mathcal{D} o \min \left(\mathcal{F}_{1}, E\right)^{+}}|-|\left(\underline{A_{2}}\right)_{\mathcal{D} o \min \left(\mathcal{F}_{1}, E\right)^{+}} \mid \\
& -\left\{\left|\left(\overline{A_{1}}\right)_{\mathcal{D} o \min \left(\mathcal{F}_{1}, E\right)^{+}} \cap\left(\overline{A_{2}}\right)_{\mathcal{D} o \min \left(\mathcal{F}_{1}, E\right)^{+}}\right|-\left|\left(\underline{A}_{1}\right)_{\mathcal{D} o \min \left(\mathcal{F}_{1}, E\right)^{+}} \cap\left(\underline{A_{2}}\right)_{\mathcal{D} o \min \left(\mathcal{F}_{1}, E\right)^{+}}\right|\right\} \\
& \leq\left|\left(\overline{A_{1}}\right)_{\mathcal{D} o \min \left(\mathcal{F}_{1}, E\right)^{+}}\right|+\left|\left(\overline{A_{2}}\right)_{\mathcal{D} o \min \left(\mathcal{F}_{1}, E\right)^{+}}\right|-\left|{\underline{\left(A_{1}\right.}}_{\mathcal{D}_{\mathcal{D} \min \left(\mathcal{F}_{1}, E\right)^{+}}}\right|-\left|{\underline{\left(A_{2}\right.}}_{\mathcal{D}_{\operatorname{lomin}\left(\mathcal{F}_{1}, E\right)^{+}}}\right| \\
& -\mu_{\mathcal{D} o \min \left(\mathcal{F}_{1}, E\right)^{+}}\left(A_{1} \cap A_{2}\right)\left|\left(\overline{A_{1}}\right)_{\mathcal{D}_{0 \min }\left(\mathcal{F}_{1}, E\right)^{+}} \cap\left(\overline{A_{2}}\right)_{\mathcal{D} o \min \left(\mathcal{F}_{1}, E\right)^{+}}\right| .
\end{aligned}
$$

Moreover by definition of the rough degree:

$$
\mu_{\mathcal{D} o \min \left(\mathcal{F}_{1}, E\right)^{+}}\left(A_{1}\right)=1-\frac{\mid \underline{\left(A_{1}\right)_{\mathcal{D} o \min \left(\mathcal{F}_{1}, E\right)^{+}} \mid}}{\left|\left(\overline{A_{1}}\right)_{\mathcal{D} o \min \left(\mathcal{F}_{1}, E\right)^{+}}\right|}
$$

and:

$$
\mu_{\mathcal{D} o \min \left(\mathcal{F}_{1}, E\right)^{+}}\left(A_{2}\right)=1-\frac{\mid\left({\underline{\left(A_{2}\right.}}_{\mathcal{D} \min \left(\mathcal{F}_{1}, E\right)^{+}} \mid\right.}{\left|\left(\overline{A_{2}}\right)_{\mathcal{D} o \min \left(\mathcal{F}_{1}, E\right)^{+}}\right|} .
$$

We obtain the following relation:

$$
\mu_{\mathcal{D} o \min \left(\mathcal{F}_{1}, E\right)^{+}}\left(A_{1}\right)\left|\left(\overline{A_{1}}\right)_{\mathcal{D} o \min \left(\mathcal{F}_{1}, E\right)^{+}}\right|=\left|\left(\overline{A_{1}}\right)_{\mathcal{D} o \min \left(\mathcal{F}_{1}, E\right)^{+}}\right|-\left|{\underline{\left(A_{1}\right.}}_{\mathcal{D}_{\min \left(\mathcal{F}_{1}, E\right)^{+}}}\right|
$$


and:

$$
\mu_{\mathcal{D} o \min \left(\mathcal{F}_{1}, E\right)^{+}}\left(A_{2}\right)\left|\left(\overline{A_{2}}\right)_{\mathcal{D} o \min \left(\mathcal{F}_{1}, E\right)^{+}}\right|=\left|\left(\overline{A_{2}}\right)_{\mathcal{D} o \min \left(\mathcal{F}_{1}, E\right)^{+}}\right|-\left|\left(\underline{A_{2}}\right)_{\mathcal{D} o \min \left(\mathcal{F}_{1}, E\right)^{+}}\right| .
$$

Therefore:

$$
\begin{aligned}
& \mu_{\mathcal{D} o \min \left(\mathcal{F}_{1}, E\right)^{+}}\left(A_{1} \cup A_{2}\right)\left|\left(\overline{A_{1}}\right)_{\mathcal{D} o \min \left(\mathcal{F}_{1}, E\right)^{+}} \cup\left(\overline{A_{2}}\right)_{\mathcal{D} o \min \left(\mathcal{F}_{1}, E\right)^{+}}\right| \\
\leq & \left\{\left|\left(\overline{A_{1}}\right)_{\mathcal{D} o \min \left(\mathcal{F}_{1}, E\right)^{+}}\right|-\left|\left(\underline{A_{1}}\right)_{\mathcal{D} o \min \left(\mathcal{F}_{1}, E\right)^{+}}\right|\right\}+\left\{\left|\left(\overline{A_{2}}\right)_{\mathcal{D} o \min \left(\mathcal{F}_{1}, E\right)^{+}}\right|-\left|\left(\underline{A_{2}}\right)_{\mathcal{D} o \min \left(\mathcal{F}_{1}, E\right)^{+}}\right|\right\} \\
& -\mu_{\mathcal{D} o \min \left(\mathcal{F}_{1}, E\right)^{+}}\left(A_{1} \cap A_{2}\right)\left|\left(\overline{A_{1}}\right)_{\mathcal{D} o \min \left(\mathcal{F}_{1}, E\right)^{+}} \cap\left(\overline{A_{2}}\right)_{\mathcal{D} o \min \left(\mathcal{F}_{1}, E\right)^{+}}\right| \\
= & \mu_{\mathcal{D} o \min \left(\mathcal{F}_{1}, E\right)^{+}}\left(A_{1}\right)\left|\left(\overline{A_{1}}\right)_{\mathcal{D} o \min \left(\mathcal{F}_{1}, E\right)^{+}}\right|+\mu_{\mathcal{D} o \min \left(\mathcal{F}_{1}, E\right)^{+}}\left(A_{2}\right)\left|\left(\overline{A_{2}}\right)_{\mathcal{D} o \min \left(\mathcal{F}_{1}, E\right)^{+}}\right| \\
& -\mu_{\mathcal{D} o \min \left(\mathcal{F}_{1}, E\right)^{+}}\left(A_{1} \cap A_{2}\right)\left|\left(\overline{A_{1}}\right)_{\mathcal{D} o \min \left(\mathcal{F}_{1}, E\right)^{+}} \cap\left(\overline{A_{2}}\right)_{\mathcal{D} o \min \left(\mathcal{F}_{1}, E\right)^{+}}\right| .
\end{aligned}
$$

(2) By definition of the rough degree $\mu_{\mathcal{D} o \min \left(\mathcal{F}_{1}, E\right)^{+}}\left(A_{1}\right)=1-\rho_{\mathcal{D} o \min \left(\mathcal{F}_{1}, E\right)^{+}}\left(A_{1}\right)$. Since:

$$
\begin{aligned}
& \mu_{\mathcal{D} o \min \left(\mathcal{F}_{1}, E\right)^{+}}\left(A_{1} \cup A_{2}\right)\left|\left(\overline{A_{1}}\right)_{\mathcal{D} o \min \left(\mathcal{F}_{1}, E\right)^{+}} \cup\left(\overline{A_{2}}\right)_{\mathcal{D} o \min \left(\mathcal{F}_{1}, E\right)^{+}}\right| \\
& \leq \mu_{\mathcal{D} o \min \left(\mathcal{F}_{1}, E\right)^{+}}\left(A_{1}\right)\left|\left(\overline{A_{1}}\right)_{\mathcal{D} o \min \left(\mathcal{F}_{1}, E\right)^{+}}\right|+\mu_{\mathcal{D} o \min \left(\mathcal{F}_{1}, E\right)^{+}}\left(A_{2}\right)\left|\left(\overline{A_{2}}\right)_{\mathcal{D} o \min \left(\mathcal{F}_{1}, E\right)^{+}}\right| \\
& -\mu_{\mathcal{D} o \min \left(\mathcal{F}_{1}, E\right)^{+}}\left(A_{1} \cap A_{2}\right)\left|\left(\overline{A_{1}}\right)_{\mathcal{D} o \min \left(\mathcal{F}_{1}, E\right)^{+}} \cap\left(\overline{A_{2}}\right)_{\mathcal{D} o \min \left(\mathcal{F}_{1}, E\right)^{+}}\right| \text {. }
\end{aligned}
$$

Therefore:

$$
\begin{aligned}
& \left\{1-\rho_{\mathcal{D} o \min \left(\mathcal{F}_{1}, E\right)^{+}}\left(A_{1} \cup A_{2}\right)\right\}\left|\left(\overline{A_{1}}\right)_{\mathcal{D} o \min \left(\mathcal{F}_{1}, E\right)^{+}} \cup\left(\overline{A_{2}}\right)_{\mathcal{D} o \min \left(\mathcal{F}_{1}, E\right)^{+}}\right| \\
\leq & \left\{1-\rho_{\mathcal{D} o \min \left(\mathcal{F}_{1}, E\right)^{+}}\left(A_{1}\right)\right\}\left|\left(\overline{A_{1}}\right)_{\mathcal{D} o \min \left(\mathcal{F}_{1}, E\right)^{+}}\right|+\left\{1-\rho_{\mathcal{D} o \min \left(\mathcal{F}_{1}, E\right)^{+}}\left(A_{2}\right)\right\}\left|\left(\overline{A_{2}}\right)_{\mathcal{D} o \min \left(\mathcal{F}_{1}, E\right)^{+}}\right| \\
& -\left\{1-\rho_{\mathcal{D} o \min \left(\mathcal{F}_{1}, E\right)^{+}}\left(A_{1} \cap A_{2}\right)\right\}\left|\left(\overline{A_{1}}\right)_{\mathcal{D} o \min \left(\mathcal{F}_{1}, E\right)^{+}} \cap\left(\overline{A_{2}}\right)_{\mathcal{D} o \min \left(\mathcal{F}_{1}, E\right)^{+}}\right| \\
= & \left|\left(\overline{A_{1}}\right)_{\mathcal{D} o \min \left(\mathcal{F}_{1}, E\right)^{+}}\right|-\rho_{\mathcal{D} o \min \left(\mathcal{F}_{1}, E\right)^{+}}\left(A_{1}\right)\left|\left(\overline{A_{1}}\right)_{\mathcal{D} o \min \left(\mathcal{F}_{1}, E\right)^{+}}\right|+\left|\left(\overline{A_{2}}\right)_{\mathcal{D} o \min \left(\mathcal{F}_{1}, E\right)^{+}}\right| \\
& -\rho_{\mathcal{D} o \min \left(\mathcal{F}_{1}, E\right)^{+}}\left(A_{2}\right)\left|\left(\overline{A_{2}}\right)_{\mathcal{D} o \min \left(\mathcal{F}_{1}, E\right)^{+}}\right|-\left|\left(\overline{A_{1}}\right)_{\mathcal{D} o \min \left(\mathcal{F}_{1}, E\right)^{+}} \cap\left(\overline{A_{2}}\right)_{\mathcal{D} o \min \left(\mathcal{F}_{1}, E\right)^{+}}\right| \\
& +\rho_{\mathcal{D} o \min \left(\mathcal{F}_{1}, E\right)^{+}}\left(A_{1} \cap A_{2}\right)\left|\left(\overline{A_{1}}\right)_{\mathcal{D} o \min \left(\mathcal{F}_{1}, E\right)^{+}} \cap\left(\overline{A_{2}}\right)_{\mathcal{D} o \min \left(\mathcal{F}_{1}, E\right)^{+}}\right| \\
= & \left|\left(\overline{A_{1}}\right)_{\mathcal{D} o \min \left(\mathcal{F}_{1}, E\right)^{+}} \cup\left(\overline{A_{2}}\right)_{\mathcal{D} o \min \left(\mathcal{F}_{1}, E\right)^{+}}\right|-\rho_{\mathcal{D} o \min \left(\mathcal{F}_{1}, E\right)^{+}}\left(A_{1}\right)\left|\left(\overline{A_{1}}\right)_{\mathcal{D} o \min \left(\mathcal{F}_{1}, E\right)^{+}}\right| \\
& -\rho_{\mathcal{D} o \min \left(\mathcal{F}_{1}, E\right)^{+}}\left(A_{2}\right)\left|\left(\overline{A_{2}}\right)_{\mathcal{D} o \min \left(\mathcal{F}_{1}, E\right)^{+}}\right| \\
& +\rho_{\mathcal{D} o \min \left(\mathcal{F}_{1}, E\right)^{+}}\left(A_{1} \cap A_{2}\right)\left|\left(\overline{A_{1}}\right)_{\mathcal{D} o \min \left(\mathcal{F}_{1}, E\right)^{+}} \cap\left(\overline{A_{2}}\right)_{\mathcal{D} o \min \left(\mathcal{F}_{1}, E\right)^{+}}\right| .
\end{aligned}
$$

By routine simplifications, we get:

$$
\begin{aligned}
& \rho_{\mathcal{D} o \min \left(\mathcal{F}_{1}, E\right)^{+}}\left(A_{1} \cup A_{2}\right)\left|\left(\overline{A_{1}}\right)_{\mathcal{D} o \min \left(\mathcal{F}_{1}, E\right)^{+}} \cup\left(\overline{A_{2}}\right)_{\mathcal{D} o \min \left(\mathcal{F}_{1}, E\right)^{+}}\right| \\
\geq & \rho_{\mathcal{D} o \min \left(\mathcal{F}_{1}, E\right)^{+}}\left(A_{1}\right)\left|\left(\overline{A_{1}}\right)_{\mathcal{D} o \min \left(\mathcal{F}_{1}, E\right)^{+}}\right|+\rho_{\mathcal{D} o \min \left(\mathcal{F}_{1}, E\right)^{+}}\left(A_{2}\right)\left|\left(\overline{A_{2}}\right)_{\mathcal{D} o \min \left(\mathcal{F}_{1}, E\right)^{+}}\right| \\
& -\rho_{\mathcal{D} o \min \left(\mathcal{F}_{1}, E\right)^{+}}\left(A_{1} \cap A_{2}\right)\left|\left(\overline{A_{1}}\right)_{\mathcal{D} o \min \left(\mathcal{F}_{1}, E\right)^{+}} \cap\left(\overline{A_{2}}\right)_{\mathcal{D} o \min \left(\mathcal{F}_{1}, E\right)^{+}}\right|
\end{aligned}
$$

Theorem 6. Let $\mathcal{I S}=(U, V, \widetilde{f})$ be the Middle East conflict situation and $A_{1}, A_{2} \subseteq U$. Then the rough degree and precision of the sets $A_{1}, A_{2}, A_{1} \cup A_{2}$, and $A_{1} \cap A_{2}$ satisfy the following relations: 
(1)

$$
\begin{aligned}
& \mu_{\mathcal{D} o \min \left(\mathcal{F}_{1}, E\right)^{-}}\left(A_{1} \cup A_{2}\right)\left|\left(\overline{A_{1}}\right)_{\mathcal{D} o \min \left(\mathcal{F}_{1}, E\right)^{-}} \cup\left(\overline{A_{2}}\right)_{\mathcal{D} o \min \left(\mathcal{F}_{1}, E\right)^{-}}\right| \\
\leq & \mu_{\mathcal{D} o \min \left(\mathcal{F}_{1}, E\right)^{-}}\left(A_{1}\right)\left|\left(\overline{A_{1}}\right)_{\mathcal{D} o \min \left(\mathcal{F}_{1}, E\right)^{-}}\right|+\mu_{\mathcal{D} o \min \left(\mathcal{F}_{1}, E\right)^{-}}\left(A_{2}\right)\left|\left(\overline{A_{2}}\right)_{\mathcal{D} o \min \left(\mathcal{F}_{1}, E\right)^{-}}\right| \\
& -\mu_{\mathcal{D} o \min \left(\mathcal{F}_{1}, E\right)^{-}}\left(A_{1} \cap A_{2}\right)\left|\left(\overline{A_{1}}\right)_{\mathcal{D} o \min \left(\mathcal{F}_{1}, E\right)^{-}} \cap\left(\overline{A_{2}}\right)_{\mathcal{D} o \min \left(\mathcal{F}_{1}, E\right)^{-}}\right| .
\end{aligned}
$$

(2)

$$
\begin{aligned}
& \rho_{\mathcal{D} o \min \left(\mathcal{F}_{1}, E\right)^{-}}\left(A_{1} \cup A_{2}\right)\left|\left(\overline{A_{1}}\right)_{\mathcal{D} o \min \left(\mathcal{F}_{1}, E\right)^{-}} \cup\left(\overline{A_{2}}\right)_{\mathcal{D} o \min \left(\mathcal{F}_{1}, E\right)^{+}}\right| \\
\geq & \rho_{\mathcal{D} o \min \left(\mathcal{F}_{1}, E\right)^{-}}\left(A_{1}\right)\left|\left(\overline{A_{1}}\right)_{\mathcal{D} o \min \left(\mathcal{F}_{1}, E\right)^{-}}\right|+\rho_{\mathcal{D} o \min \left(\mathcal{F}_{1}, E\right)^{-}}\left(A_{2}\right)\left|\left(\overline{A_{2}}\right)_{\mathcal{D} o \min \left(\mathcal{F}_{1}, E\right)^{-}}\right| \\
& -\rho_{\mathcal{D} o \min \left(\mathcal{F}_{1}, E\right)^{-}}\left(A_{1} \cap A_{2}\right)\left|\left(\overline{A_{1}}\right)_{\mathcal{D}_{\min }\left(\mathcal{F}_{1}, E\right)^{-}} \cap\left(\overline{A_{2}}\right)_{\mathcal{D} o \min \left(\mathcal{F}_{1}, E\right)^{-}}\right|
\end{aligned}
$$

Proof. The proof is similar to the proof of Theorem 5.

Definition 7. Let $\mathcal{I} \mathcal{S}=(U, V, \widetilde{f})$ be the Middle East conflict situation. For any set $A_{1} \subseteq U$, the approximate quality $\gamma_{\mathcal{D} o \min \left(\mathcal{F}_{1}, E\right)^{+}}\left(A_{1}\right)$ and $\gamma_{\mathcal{D} o \min \left(\mathcal{F}_{1}, E\right)^{-}}\left(A_{1}\right)$ of $A_{1}$ about $\operatorname{Domin}\left(\mathcal{F}_{1}, E\right)^{+}$and Do $\min \left(\mathcal{F}_{1}, E\right)^{-}$are defined as follows:

$$
\gamma_{\mathcal{D} o \min \left(\mathcal{F}_{1}, E\right)^{+}}\left(A_{1}\right)=\frac{\left|\left(\underline{A}_{1}\right)_{\mathcal{D} o \min \left(\mathcal{F}_{1}, E\right)^{+}}\right|}{|U|} \text { and } \gamma_{\mathcal{D o m i n}\left(\mathcal{F}_{1}, E\right)^{-}}\left(A_{1}\right)=\frac{\left|\left(\underline{A}_{1}\right)_{\mathcal{D o m i n}\left(\mathcal{F}_{1}, E\right)^{-}}\right|}{|U|} .
$$

Clearly, $0 \leq \gamma_{\mathcal{D} o \min \left(\mathcal{F}_{1}, E\right)^{+}}\left(A_{1}\right) \leq 1$ and $0 \leq \gamma_{\mathcal{D o m i n}\left(\mathcal{F}_{1}, E\right)^{-}}\left(A_{1}\right) \leq 1$.

The following theorem illustrate the relationship between the rough degree and approximate quality with respect to the intersection and union of sets $A_{1}$ and $A_{2}$ on the universe $U$.

Theorem 7. Let $\mathcal{I} \mathcal{S}=(U, V, \widetilde{f})$ be the Middle East conflict situation and $A_{1}, A_{2} \subseteq U$. Then the rough degree and approximate quality of the sets $A_{1}, A_{2}, A_{1} \cup A_{2}$, and $A_{1} \cap A_{2}$ satisfy the following relations:

(1)

$$
\begin{aligned}
& \mu_{\mathcal{D} o \min \left(\mathcal{F}_{1}, E\right)^{+}}\left(A_{1} \cup A_{2}\right)\left|\left(\overline{A_{1}}\right)_{\mathcal{D} o \min \left(\mathcal{F}_{1}, E\right)^{+}} \cup\left(\overline{A_{2}}\right)_{\mathcal{D} o \min \left(\mathcal{F}_{1}, E\right)^{+}}\right| \\
& \leq\left|\left(\overline{A_{1}}\right)_{\mathcal{D} o \min \left(\mathcal{F}_{1}, E\right)^{+}}\right|+\left|\left(\overline{A_{2}}\right)_{\mathcal{D} o \min \left(\mathcal{F}_{1}, E\right)^{+}}\right|-|U|\left\{\gamma_{\mathcal{D} o \min \left(\mathcal{F}_{1}, E\right)^{+}}\left(A_{1}\right)+\gamma_{\mathcal{D} o \min \left(\mathcal{F}_{1}, E\right)^{+}}\left(A_{2}\right)\right\} \\
& -\mu_{\mathcal{D} o \min \left(\mathcal{F}_{1}, E\right)^{+}}\left(A_{1} \cap A_{2}\right)\left|\left(\overline{A_{1}}\right)_{\mathcal{D} o \min \left(\mathcal{F}_{1}, E\right)^{+}} \cap\left(\overline{A_{2}}\right)_{\mathcal{D} o \min \left(\mathcal{F}_{1}, E\right)^{+}}\right| \text {. }
\end{aligned}
$$

(2)

$$
\begin{aligned}
& \mu_{\mathcal{D} o \min \left(\mathcal{F}_{1}, E\right)^{-}}\left(A_{1} \cup A_{2}\right)\left|\left(\overline{A_{1}}\right)_{\mathcal{D} o \min \left(\mathcal{F}_{1}, E\right)^{-}} \cup\left(\overline{A_{2}}\right)_{\mathcal{D} o \min \left(\mathcal{F}_{1}, E\right)^{-}}\right| \\
\leq & \left|\left(\overline{A_{1}}\right)_{\mathcal{D} o \min \left(\mathcal{F}_{1}, E\right)^{-}}\right|+\left|\left(\overline{A_{2}}\right)_{\mathcal{D} o \min \left(\mathcal{F}_{1}, E\right)^{-}}\right|-|U|\left\{\gamma_{\mathcal{D} o \min \left(\mathcal{F}_{1}, E\right)^{-}}\left(A_{1}\right)+\gamma_{\mathcal{D} o \min \left(\mathcal{F}_{1}, E\right)^{-}}\left(A_{2}\right)\right\} \\
& -\mu_{\mathcal{D} o \min \left(\mathcal{F}_{1}, E\right)^{-}}\left(A_{1} \cap A_{2}\right)\left|\left(\overline{A_{1}}\right)_{\mathcal{D} o \min \left(\mathcal{F}_{1}, E\right)^{-}} \cap\left(\overline{A_{2}}\right)_{\mathcal{D} o \min \left(\mathcal{F}_{1}, E\right)^{-}}\right| .
\end{aligned}
$$

Proof. The proof is similar to the proof of Theorem 5.

The following theorem highlights the relationship between the approximate precision and approximate quality in regards to the intersection and union of two sets. 
Theorem 8. Let $\mathcal{I S}=(U, V, \widetilde{f})$ be the Middle East conflict situation and $A_{1}, A_{2} \subseteq U$. Then the approximate quality and precision of the sets $A_{1}, A_{2}, A_{1} \cup A_{2}$, and $A_{1} \cap A_{2}$ satisfy the following relations: (1)

$$
\begin{aligned}
& \rho_{\mathcal{D} o \min \left(\mathcal{F}_{1}, E\right)^{+}}\left(A_{1} \cup A_{2}\right)\left|\left(\overline{A_{1}}\right)_{\mathcal{D} o \min \left(\mathcal{F}_{1}, E\right)^{+}} \cup\left(\overline{A_{2}}\right)_{\mathcal{D} o \min \left(\mathcal{F}_{1}, E\right)^{+}}\right| \\
\geq & |U|\left(\gamma_{\mathcal{D} o \min \left(\mathcal{F}_{1}, E\right)^{+}}\left(A_{1}\right)+\gamma_{\mathcal{D} o \min \left(\mathcal{F}_{1}, E\right)^{+}}\left(A_{2}\right)\right) \\
& -\rho_{\mathcal{D} o \min \left(\mathcal{F}_{1}, E\right)^{+}}\left(A_{1} \cap A_{2}\right)\left|\left(\overline{A_{1}}\right)_{\mathcal{D} o \min \left(\mathcal{F}_{1}, E\right)^{+}} \cap\left(\overline{A_{2}}\right)_{\mathcal{D} o \min \left(\mathcal{F}_{1}, E\right)^{+}}\right| .
\end{aligned}
$$

(2)

$$
\begin{aligned}
& \rho_{\mathcal{D} o \min \left(\mathcal{F}_{1}, E\right)^{-}}\left(A_{1} \cup A_{2}\right)\left|\left(\overline{A_{1}}\right)_{\mathcal{D} o \min \left(\mathcal{F}_{1}, E\right)^{-}} \cup\left(\overline{A_{2}}\right)_{\mathcal{D} o \min \left(\mathcal{F}_{1}, E\right)^{-}}\right| \\
\geq & |U|\left(\gamma_{\mathcal{D} o \min \left(\mathcal{F}_{1}, E\right)^{-}}\left(A_{1}\right)+\gamma_{\mathcal{D} o \min \left(\mathcal{F}_{1}, E\right)^{-}}\left(A_{2}\right)\right) \\
& -\rho_{\mathcal{D} o \min \left(\mathcal{F}_{1}, E\right)^{-}}\left(A_{1} \cap A_{2}\right)\left|\left(\overline{A_{1}}\right)_{\mathcal{D} o \min \left(\mathcal{F}_{1}, E\right)^{-}} \cap\left(\overline{A_{2}}\right)_{\mathcal{D} o \min \left(\mathcal{F}_{1}, E\right)^{-}}\right| .
\end{aligned}
$$

Proof. The proof is similar to the proof of Theorem 5.

Definition 8. Let $\mathcal{I} \mathcal{S}=(U, V, \widetilde{f})$ be the Middle East conflict situation. For any subset $A_{1}$ of $U$, the approximate accuracies $\varsigma_{[u]_{\mathcal{D} o \min \left(\mathcal{F}_{1}, E\right)}^{+}}\left(A_{1}\right)$ and $\varsigma_{[u]_{\mathcal{D} o \min \left(\mathcal{F}_{1}, E\right)}^{-}}\left(A_{1}\right)$ of $A_{1}$ about $[u]_{\mathcal{D} o \min \left(\mathcal{F}_{1}, E\right)}^{+}$and $[u]_{\mathcal{D} o \min \left(\mathcal{F}_{1}, E\right)}^{-}$, respectively, are defined by:

$$
\varsigma_{[u]_{\mathcal{D} o \min \left(\mathcal{F}_{1}, E\right)}^{+}}\left(A_{1}\right)=\frac{\left|[u]_{\mathcal{D} o \min \left(\mathcal{F}_{1}, E\right)}^{+} \cap A_{1}\right|}{\left|[u]_{\mathcal{D} o \min \left(\mathcal{F}_{1}, E\right)}^{+}\right|}
$$

and:

$$
\varsigma_{[u]_{\mathcal{D} o \min \left(\mathcal{F}_{1}, E\right)}^{-}}\left(A_{1}\right)=\frac{\left|[u]_{\mathcal{D} o \min \left(\mathcal{F}_{1}, E\right)}^{-} \cap A_{1}\right|}{\left|[u]_{\mathcal{D} o \min \left(\mathcal{F}_{1}, E\right)}^{-}\right|} .
$$

It is routine to verify that $0 \leq \varsigma_{[u]_{\mathcal{D} o \min \left(\mathcal{F}_{1}, E\right)}^{+}}\left(A_{1}\right) \leq 1$ and $0 \leq \varsigma_{[u]_{\mathcal{D} o \min \left(\mathcal{F}_{1}, E\right)}^{-}}\left(A_{1}\right) \leq 1$. For any $A_{1}, A_{2} \subseteq U$, the following properties hold:

(1) $S_{[u]_{\mathcal{D} o \min \left(\mathcal{F}_{1}, E\right)}^{+}}\left(A_{1}\right)=1$ if and only if $u \in\left(\underline{A_{1}}\right)_{\mathcal{D} o \min \left(\mathcal{F}_{1}, E\right)^{+}}$，

(2) $\varsigma_{[u]_{\mathcal{D} o \min \left(\mathcal{F}_{1}, E\right)}^{+}}\left(A_{1}\right)=0$ if and only if $u \in\left(\underline{A_{1}^{c}}\right)_{\mathcal{D} o \min \left(\mathcal{F}_{1}, E\right)^{+}}$,

(3) $\varsigma_{[u]_{\mathcal{D} o \min \left(\mathcal{F}_{1}, E\right)}^{+}}\left(A_{1} \cup A_{2}\right)=\varsigma_{[u]_{\mathcal{D} o \min \left(\mathcal{F}_{1}, E\right)}^{+}}\left(A_{1}\right)+\varsigma_{[u]_{\mathcal{D} o \min \left(\mathcal{F}_{1}, E\right)}^{+}}\left(A_{2}\right)-\varsigma_{[u]_{\mathcal{D} o \min \left(\mathcal{F}_{1}, E\right)}^{+}}\left(A_{1} \cap A_{2}\right)$,

(4) $\varsigma_{[u]_{\mathcal{D} o \min \left(\mathcal{F}_{1}, E\right)}^{+}}\left(A_{1}\right)=1-\varsigma_{[u]_{\mathcal{D} o \min \left(\mathcal{F}_{1}, E\right)}^{+}}\left(A_{1}\right)$,

(5) If $A_{1} \subseteq A_{2}$, then $\varsigma_{[u]_{\mathcal{D} o \min \left(\mathcal{F}_{1}, E\right)}^{+}}\left(A_{1}\right) \leq \varsigma_{[u]_{\mathcal{D} o \min \left(\mathcal{F}_{1}, E\right)}^{+}}\left(A_{2}\right)$,

(6) $\varsigma_{[u]_{\mathcal{D} o \min \left(\mathcal{F}_{1}, E\right)}^{+}}(\varnothing)=0$ and $\varsigma_{[u]_{\mathcal{D} o \min \left(\mathcal{F}_{1}, E\right)}^{+}}(U)=1$, for every $u \in U$, and

(7) $\varsigma_{[u]_{\mathcal{D} o \min \left(\mathcal{F}_{1}, E\right)}^{+}}\left(A_{1}\right)+\varsigma_{[u]_{\mathcal{D} o \min \left(\mathcal{F}_{1}, E\right)}^{+}}\left(A_{1}\right)=1$.

Using the soft preference relation we give an affirmative response to the aforesaid drawbacks of Sun et al.'s technique:

Let $\mathcal{I S}=(U, V, \widetilde{f})$ be the Middle East conflict situation and $E \subseteq V$ be a feasible strategy for resolving the conflict situation. Then for any $A_{1}=\{u \in U: f(u, a) \neq 0, \forall a \in E\} \subseteq U$, denote:

The agreement subset $=A_{S}=\left(\underline{A}_{1}\right)_{\mathcal{D} o \min \left(\mathcal{F}_{1}, E\right)^{+}}-\left(\underline{A_{1}}\right)_{\mathcal{D} o \min \left(\mathcal{F}_{1}, E\right)^{-}}$, 
The disagreement subset $=D_{S}=\left(\underline{A_{1}}\right)_{\mathcal{D} o \min \left(\mathcal{F}_{1}, E\right)^{-}}-\left(\underline{A_{1}}\right)_{\mathcal{D o}_{\min \left(\mathcal{F}_{1}, E\right)^{+}}}$and The neutral subset $=N_{S}=U-\left(A_{S} \cup D_{S}\right)$.

The relationships between the agreement, disagreement, and neutral subsets are:
(i) $A_{S} \cap D_{S}=\varnothing$
(ii) $A_{S} \cap N_{S}=\varnothing$
(iii) $D_{S} \cap N_{S}=\varnothing$
(iv) $A_{S} \cup D_{S} \cup N_{S}=U$.

Example 2. (Continued from Example 1) Consider the Information Table 1 for the Middle East conflict analysis. If $E_{1}=\left\{a_{2}, a_{3}, a_{5}\right\} \subseteq V$, then the soft preference relation $F: E_{1} \rightarrow P(U \times U)$ is defined by:

$$
\begin{gathered}
F\left(a_{2}\right)=\left\{\begin{array}{r}
\left(u_{1}, u_{1}\right),\left(u_{2}, u_{2}\right),\left(u_{3}, u_{3}\right),\left(u_{4}, u_{4}\right),\left(u_{5}, u_{5}\right),\left(u_{6}, u_{6}\right),\left(u_{1}, u_{2}\right), \\
\left(u_{1}, u_{3}\right),\left(u_{1}, u_{4}\right),\left(u_{1}, u_{5}\right),\left(u_{1}, u_{6}\right),\left(u_{2}, u_{3}\right),\left(u_{2}, u_{4}\right),\left(u_{2}, u_{5}\right), \\
\left(u_{3}, u_{4}\right),\left(u_{3}, u_{5}\right),\left(u_{4}, u_{3}\right),\left(u_{4}, u_{5}\right),\left(u_{5}, u_{3}\right),\left(u_{5}, u_{4}\right),\left(u_{6}, u_{1}\right), \\
\left(u_{6}, u_{2}\right),\left(u_{6}, u_{3}\right),\left(u_{6}, u_{4}\right),\left(u_{6}, u_{5}\right)
\end{array}\right\}, \\
F\left(a_{3}\right)=\left\{\begin{array}{r}
\left(u_{1}, u_{1}\right),\left(u_{2}, u_{2}\right),\left(u_{3}, u_{3}\right),\left(u_{4}, u_{4}\right),\left(u_{5}, u_{5}\right),\left(u_{6}, u_{6}\right),\left(u_{1}, u_{2}\right), \\
\left(u_{1}, u_{3}\right),\left(u_{1}, u_{4}\right),\left(u_{1}, u_{5}\right),\left(u_{1}, u_{6}\right),\left(u_{2}, u_{3}\right),\left(u_{2}, u_{4}\right),\left(u_{2}, u_{5}\right), \\
\left(u_{2}, u_{6}\right),\left(u_{3}, u_{2}\right),\left(u_{3}, u_{4}\right),\left(u_{3}, u_{5}\right),\left(u_{3}, u_{6}\right),\left(u_{4}, u_{2}\right),\left(u_{4}, u_{3}\right), \\
\left(u_{4}, u_{5}\right),\left(u_{4}, u_{6}\right),\left(u_{5}, u_{2}\right),\left(u_{5}, u_{3}\right),\left(u_{5}, u_{4}\right),\left(u_{5}, u_{6}\right),\left(u_{6}, u_{2}\right), \\
\left(u_{6}, u_{2}\right),\left(u_{6}, u_{4}\right),\left(u_{6}, u_{5}\right)
\end{array}\right\}, \\
F\left(a_{5}\right)=\left\{\begin{array}{r}
\left(u_{1}, u_{1}\right),\left(u_{2}, u_{2}\right),\left(u_{3}, u_{3}\right),\left(u_{4}, u_{4}\right),\left(u_{5}, u_{5}\right),\left(u_{6}, u_{6}\right),\left(u_{1}, u_{2}\right), \\
\left(u_{1}, u_{3}\right),\left(u_{1}, u_{4}\right),\left(u_{1}, u_{5}\right),\left(u_{1}, u_{6}\right),\left(u_{2}, u_{4}\right),\left(u_{2}, u_{5}\right),\left(u_{3}, u_{2}\right), \\
\left(u_{3}, u_{4}\right),\left(u_{3}, u_{5}\right),\left(u_{4}, u_{2}\right),\left(u_{4}, u_{5}\right),\left(u_{5}, u_{2}\right),\left(u_{5}, u_{4}\right),\left(u_{6}, u_{1}\right), \\
\left(u_{6}, u_{2}\right),\left(u_{6}, u_{3}\right),\left(u_{6}, u_{4}\right),\left(u_{6}, u_{5}\right)
\end{array}\right\} .
\end{gathered}
$$

The soft dominance relation is given by:

$$
\mathcal{D} o \min \left(\mathcal{F}_{1}, E_{1}\right)=\left\{\begin{array}{c}
\left(u_{1}, u_{1}\right),\left(u_{2}, u_{2}\right),\left(u_{3}, u_{3}\right),\left(u_{4}, u_{4}\right),\left(u_{5}, u_{5}\right),\left(u_{6}, u_{6}\right), \\
\left(u_{1}, u_{2}\right),\left(u_{1}, u_{3}\right),\left(u_{1}, u_{4}\right),\left(u_{1}, u_{5}\right),\left(u_{1}, u_{6}\right),\left(u_{2}, u_{4}\right), \\
\left(u_{2}, u_{5}\right),\left(u_{3}, u_{4}\right),\left(u_{3}, u_{5}\right),\left(u_{4}, u_{5}\right),\left(u_{5}, u_{4}\right),\left(u_{6}, u_{2}\right), \\
\left(u_{6}, u_{3}\right),\left(u_{6}, u_{4}\right),\left(u_{6}, u_{5}\right)
\end{array}\right\} .
$$

The dominance classes from $\mathcal{D}$ o $\min \left(\mathcal{F}_{1}, E_{1}\right)$ are given in the Table 2.

Table 2. The dominating classes $\left[u_{i}\right]_{\mathcal{D} o \min \left(\mathcal{F}_{1}, E_{1}\right)}^{+}$and dominated classes $\left[u_{i}\right]_{\mathcal{D} o \min \left(\mathcal{F}_{1}, E_{1}\right)}^{-}$.

\begin{tabular}{lll}
\hline$U$ & {$\left[u_{i}\right]_{\mathcal{D} o \min \left(\mathcal{F}_{1}, E\right)}^{+}$} & {$\left[u_{i}\right]_{\mathcal{D} \boldsymbol{o} \min \left(\mathcal{F}_{1}, E\right)}^{-}$} \\
\hline$u_{1}$ & $\left\{u_{1}\right\}$ & $\left\{u_{1}, u_{2}, u_{3}, u_{4}, u_{5}, u_{6}\right\}$ \\
$u_{2}$ & $\left\{u_{1}, u_{2}, u_{6}\right\}$ & $\left\{u_{2}, u_{5}\right\}$ \\
$u_{3}$ & $\left\{u_{1}, u_{3}, u_{6}\right\}$ & $\left\{u_{3}, u_{4}, u_{5}\right\}$ \\
$u_{4}$ & $\left\{u_{1}, u_{2}, u_{3}, u_{4}, u_{5}, u_{6}\right\}$ & $\left\{u_{4}, u_{5}\right\}$ \\
$u_{5}$ & $\left\{u_{1}, u_{2}, u_{3}, u_{4}, u_{5}, u_{6}\right\}$ & $\left\{u_{4}, u_{5}\right\}$ \\
$u_{6}$ & $\left\{u_{1}, u_{6}\right\}$ & $\left\{u_{2}, u_{3}, u_{4}, u_{5}, u_{6}\right\}$ \\
\hline
\end{tabular}

Let $A_{1}=\left\{u_{1}, u_{4}, u_{5}, u_{6}\right\} \subseteq U$. Then $\left(\underline{A}_{1}\right)_{\mathcal{D o m i n}\left(\mathcal{F}_{1}, E_{1}\right)^{+}}=\left\{u_{1}, u_{6}\right\}$ and $\left(\underline{A}_{1}\right)_{\mathcal{D o m i n}\left(\mathcal{F}_{1}, E_{1}\right)^{-}}=\left\{u_{4}, u_{5}\right\}$.

By using the aforesaid definition, the agreement subset $=\left\{u_{1}, u_{6}\right\}$, disagreement subset $=\left\{u_{4}, u_{5}\right\}$ and neutral subset $=\left\{u_{2}, u_{3}\right\}$. So we say that for the feasible strategy $E_{1}=\left\{a_{2}, a_{3}, a_{5}\right\} \subseteq V$ of the conflict situation, the agents $u_{4}$ and $u_{5}$ disagree with $E_{1}=\left\{a_{2}, a_{3}, a_{5}\right\}$, the agents $u_{1}$ and $u_{6}$ agree with $E_{1}=\left\{a_{2}, a_{3}, a_{5}\right\}$ and the attitude of the agents $u_{2}$ and $u_{3}$ are neutral for the feasible strategy $E_{1}=\left\{a_{2}, a_{3}, a_{5}\right\}$. In order to provide some information to decision makers, it is more pertinent to distinguish the agreement and disagreement subsets 
of agents rather than to distinguish the neutral subset attitudes of the conflict situation. Hence, we disclose information about agreement and disagreement for any feasible strategy in the conflict situation by the agreement and disagreement subsets. Also, we ascertain the feasible consensus strategy $E_{1}=\left\{a_{2}, a_{3}, a_{5}\right\} \subseteq V$ for the conflict situation by choosing the maximum cardinality of the agreement subset. Thus, we have answered the question "How can a feasible consensus strategy be found?" by using the agreement subset.

Example 3. (Continued from Example 1) Let the feasible strategy $E_{2}=\left\{a_{3}\right\} \subseteq V$. The soft dominance relation is given by:

$$
\mathcal{D} o \min \left(\mathcal{F}_{1}, E_{2}\right)=\left\{\begin{array}{c}
\left(u_{1}, u_{1}\right),\left(u_{2}, u_{2}\right),\left(u_{3}, u_{3}\right),\left(u_{4}, u_{4}\right),\left(u_{5}, u_{5}\right),\left(u_{6}, u_{6}\right),\left(u_{1}, u_{2}\right), \\
\left(u_{1}, u_{3}\right),\left(u_{1}, u_{4}\right),\left(u_{1}, u_{5}\right),\left(u_{1}, u_{6}\right),\left(u_{2}, u_{3}\right),\left(u_{2}, u_{4}\right),\left(u_{2}, u_{5}\right), \\
\left(u_{2}, u_{6}\right),\left(u_{3}, u_{2}\right),\left(u_{3}, u_{4}\right),\left(u_{3}, u_{5}\right),\left(u_{3}, u_{6}\right),\left(u_{4}, u_{2}\right),\left(u_{4}, u_{3}\right), \\
\left(u_{4}, u_{5}\right),\left(u_{4}, u_{6}\right),\left(u_{5}, u_{2}\right),\left(u_{5}, u_{3}\right),\left(u_{5}, u_{4}\right),\left(u_{5}, u_{6}\right),\left(u_{6}, u_{2}\right), \\
\left(u_{6}, u_{2}\right),\left(u_{6}, u_{4}\right),\left(u_{6}, u_{5}\right)
\end{array}\right\} .
$$

The dominance classes from $\mathcal{D}$ o $\min \left(\mathcal{F}_{1}, E_{2}\right)$ are given in Table 3.

Table 3. The dominating classes $\left[u_{i}\right]_{\mathcal{D} o \min \left(\mathcal{F}_{1}, E_{2}\right)}^{+}$and the dominated classes $\left[u_{i}\right]_{\mathcal{D} o \min \left(\mathcal{F}_{1}, E_{2}\right)}^{-}$.

\begin{tabular}{lll}
\hline$U$ & {$\left[u_{i}\right]_{\mathcal{D} o \min \left(\mathcal{F}_{1}, E_{2}\right)}^{+}$} & {$\left[u_{i}\right]_{\mathcal{D} \boldsymbol{o} \min \left(\mathcal{F}_{1}, E_{2}\right)}^{-}$} \\
\hline$u_{1}$ & $\left\{u_{1}\right\}$ & $\left\{u_{1}, u_{2}, u_{3}, u_{4}, u_{5}, u_{6}\right\}$ \\
$u_{2}$ & $\left\{u_{1}, u_{2}, u_{3}, u_{4}, u_{5}, u_{6}\right\}$ & $\left\{u_{2}, u_{3}, u_{4}, u_{5}, u_{6}\right\}$ \\
$u_{3}$ & $\left\{u_{1}, u_{2}, u_{3}, u_{4}, u_{5}, u_{6}\right\}$ & $\left\{u_{2}, u_{3}, u_{4}, u_{5}, u_{6}\right\}$ \\
$u_{4}$ & $\left\{u_{1}, u_{2}, u_{3}, u_{4}, u_{5}, u_{6}\right\}$ & $\left\{u_{2}, u_{3}, u_{4}, u_{5}, u_{6}\right\}$ \\
$u_{5}$ & $\left\{u_{1}, u_{2}, u_{3}, u_{4}, u_{5}, u_{6}\right\}$ & $\left\{u_{2}, u_{3}, u_{4}, u_{5}, u_{6}\right\}$ \\
$u_{6}$ & $\left\{u_{1}, u_{2}, u_{3}, u_{4}, u_{5}, u_{6}\right\}$ & $\left\{u_{2}, u_{3}, u_{4}, u_{5}, u_{6}\right\}$ \\
\hline
\end{tabular}

Let $A_{1}=\left\{u_{1}, u_{2}, u_{3}, u_{4}, u_{5}, u_{6}\right\}$. Then $\left(\underline{A_{1}}\right)_{\mathcal{D} o \min \left(\mathcal{F}_{1}, E_{2}\right)^{+}}=\left\{u_{1}, u_{2}, u_{3}, u_{4}, u_{5}, u_{6}\right\}$ and $\left(\underline{A_{1}}\right)_{\mathcal{D o m i n}\left(\mathcal{F}_{1}, E_{2}\right)^{-}}=\left\{u_{1}, u_{2}, u_{3}, u_{4}, u_{5}, u_{6}\right\}$. Now

$A_{S}=\varnothing, D_{S}=\varnothing$, and $N_{S}=\left\{u_{1}, u_{2}, u_{3}, u_{4}, u_{5}, u_{6}\right\}$.

Example 4. (Continued from Example 1) (iii) For the feasible strategy $E_{3}=\left\{A_{1}, a_{3}\right\} \subseteq V$. The soft dominance relation is given by:

$$
\mathcal{D} o \min \left(\mathcal{F}_{1}, E_{3}\right)=\left\{\begin{array}{c}
\left(u_{1}, u_{1}\right),\left(u_{2}, u_{2}\right),\left(u_{3}, u_{3}\right),\left(u_{4}, u_{4}\right),\left(u_{5}, u_{5}\right),\left(u_{6}, u_{6}\right), \\
\left(u_{2}, u_{3}\right),\left(u_{2}, u_{4}\right),\left(u_{2}, u_{5}\right),\left(u_{2}, u_{6}\right),\left(u_{3}, u_{2}\right),\left(u_{3}, u_{4}\right), \\
\left(u_{3}, u_{5}\right),\left(u_{3}, u_{6}\right),\left(u_{4}, u_{6}\right),\left(u_{5}, u_{2}\right),\left(u_{5}, u_{3}\right),\left(u_{5}, u_{4}\right), \\
\left(u_{5}, u_{6}\right),\left(u_{6}, u_{4}\right)
\end{array}\right\} .
$$

The dominance classes from $\mathcal{D} o \min \left(\mathcal{F}_{1}, E_{3}\right)$ are given in Table 4.

Table 4. The dominating classes $\left[u_{i}\right]_{\mathcal{D} o \min \left(\mathcal{F}_{1}, E_{3}\right)}^{+}$and the dominated classes $\left[u_{i}\right]_{\mathcal{D} o \min \left(\mathcal{F}_{1}, E_{3}\right)}^{-}$.

\begin{tabular}{lll}
\hline$U$ & {$\left[u_{i}\right]_{\mathcal{D} o \min \left(\mathcal{F}_{1}, E_{3}\right)}^{+}$} & {$\left[u_{i}\right]_{\mathcal{D} o \min \left(\mathcal{F}_{1}, E_{3}\right)}^{-}$} \\
\hline$u_{1}$ & $\left\{u_{1}\right\}$ & $\left\{u_{1}\right\}$ \\
$u_{2}$ & $\left\{u_{2}, u_{3}, u_{5}\right\}$ & $\left\{u_{2}, u_{3}, u_{4}, u_{5}, u_{6}\right\}$ \\
$u_{3}$ & $\left\{u_{2}, u_{3}, u_{5}\right\}$ & $\left\{u_{2}, u_{3}, u_{4}, u_{5}, u_{6}\right\}$ \\
$u_{4}$ & $\left\{u_{2}, u_{3}, u_{4}, u_{5}, u_{6}\right\}$ & $\left\{u_{4}, u_{6}\right\}$ \\
$u_{5}$ & $\left\{u_{2}, u_{3}, u_{5}\right\}$ & $\left\{u_{2}, u_{3}, u_{4}, u_{5}, u_{6}\right\}$ \\
$u_{6}$ & $\left\{u_{1}, u_{2}, u_{3}, u_{4}, u_{5}, u_{6}\right\}$ & $\left\{u_{4}, u_{6}\right\}$ \\
\hline
\end{tabular}

Let $A_{1}=\left\{u_{1}, u_{2}, u_{3}, u_{5}\right\}$. Then $\left(\underline{A_{1}}\right)_{\mathcal{D} o \min \left(\mathcal{F}_{1}, E_{3}\right)^{+}}=\left\{u_{1}, u_{2}, u_{3}, u_{5},\right\}$ and $\left(\underline{A_{1}}\right)_{\mathcal{D o m i n}\left(\mathcal{F}_{1}, E_{3}\right)^{-}}=\left\{u_{1}\right\}$. $A_{S}=\left\{u_{2}, u_{3}, u_{5}\right\}, D_{S}=\varnothing$, and $N_{S}=\left\{u_{1}, u_{4}, u_{6}\right\}$. 
Example 5. (Continued from Example 1) (iv) Let the feasible strategy $E_{4}=\left\{a_{1}, a_{2}, a_{3}, a_{4}, a_{5}\right\} \subseteq V$. The soft dominance relation is given by:

$$
\mathcal{D} o \min \left(\mathcal{F}_{1}, E_{4}\right)=\left\{\begin{array}{c}
\left(u_{1}, u_{1}\right),\left(u_{2}, u_{2}\right),\left(u_{3}, u_{3}\right),\left(u_{4}, u_{4}\right),\left(u_{5}, u_{5}\right),\left(u_{6}, u_{6}\right), \\
\left(u_{2}, u_{5}\right),\left(u_{3}, u_{5}\right),\left(u_{6}, u_{4}\right)
\end{array}\right\} .
$$

The dominance classes from $\mathcal{D} o \min \left(\mathcal{F}_{1}, E_{4}\right)$ are given in the Table 5.

Table 5. The dominating classes $\left[u_{i}\right]_{\mathcal{D} o \min \left(\mathcal{F}_{1}, E_{4}\right)}^{+}$and the dominated classes $\left[u_{i}\right]_{\mathcal{D} o \min \left(\mathcal{F}_{1}, E_{4}\right)}^{-}$.

\begin{tabular}{lll}
\hline$U$ & {$\left[u_{i}\right]_{\mathcal{D} o \min \left(\mathcal{F}_{1}, E_{4}\right)}^{+}$} & {$\left[u_{i}\right]_{\mathcal{D} o \min \left(\mathcal{F}_{1}, E_{4}\right)}^{-}$} \\
\hline$u_{1}$ & $\left\{u_{1}\right\}$ & $\left\{u_{1}\right\}$ \\
$u_{2}$ & $\left\{u_{2}\right\}$ & $\left\{u_{2}, u_{5}\right\}$ \\
$u_{3}$ & $\left\{u_{3}\right\}$ & $\left\{u_{3}, u_{5}\right\}$ \\
$u_{4}$ & $\left\{u_{4}, u_{6}\right\}$ & $\left\{u_{4}\right\}$ \\
$u_{5}$ & $\left\{u_{2}, u_{3}, u_{5}\right\}$ & $\left\{u_{5}\right\}$ \\
$u_{6}$ & $\left\{u_{6}\right\}$ & $\left\{u_{4}, u_{6}\right\}$ \\
\hline
\end{tabular}

Let $A_{1}=\left\{u_{1}, u_{5}\right\}$. Then by our proposed technique, the agreement subset $=\varnothing$, the disagreement subset $=\left\{u_{5}\right\}$ and the neutral subset $=\left\{u_{1}, u_{2}, u_{3}, u_{6}\right\}$ depict the real spirit of the conflict situation, while in this case Sun et al.'s technique concluded that the conflict table under consideration is neutral.

Next for any issue $a_{i} \in V$, we can easily obtain attitude information for every agent with respect to issue $a_{i} \in V$ by using $F\left(a_{i}\right)$ which is a preference relation. $F\left(a_{i}\right)$ is more informative than the two set valued mappings of Reference [4]. Thus, we answered the question "What are the intrinsic conflict reasons?" by using $F\left(a_{i}\right)$ where $a_{i} \in V$. In conclusion, we replied to Deja's questions in the best way.

Now we present another methodology for determining the feasible consensus strategy of the conflict situation. The other representation of the Middle East conflict information table is given in Table 6.

Table 6. Information table for the Middle East conflict.

\begin{tabular}{lllllll}
\hline $\boldsymbol{V} \backslash \boldsymbol{U}$ & $\boldsymbol{u}_{\mathbf{1}}$ & $\boldsymbol{u}_{\mathbf{2}}$ & $\boldsymbol{u}_{\mathbf{3}}$ & $\boldsymbol{u}_{\mathbf{4}}$ & $\boldsymbol{u}_{\mathbf{5}}$ & $\boldsymbol{u}_{\mathbf{6}}$ \\
\hline$a_{1}$ & - & + & + & 0 & + & 0 \\
$a_{2}$ & + & 0 & - & - & - & + \\
$a_{3}$ & + & - & - & - & - & - \\
$a_{4}$ & + & - & - & 0 & - & 0 \\
$a_{5}$ & + & - & 0 & - & - & + \\
\hline
\end{tabular}

According to Sun et al. [4], let $g=\left\{g^{+}, g^{-}\right\}$be a set valued mapping from $V$ to $U$, where:

$$
\begin{aligned}
& g^{+}: V \rightarrow \mathcal{P}(U), g^{+}(a)=\{u \in U \mid g(a, u)=+\} \text { for all } a \in V, \\
& g^{-}: V \rightarrow \mathcal{P}(U), g^{-}(a)=\{u \in U \mid g(a, u)=-\} \text { for all } a \in V .
\end{aligned}
$$

Now for any combination of agents $A_{1} \in \mathcal{P}(U)$ in a conflict situation, the approximations are defined as follows:

$$
\begin{gathered}
\underline{a p r}_{g}^{+}\left(A_{1}\right)=\left\{a \in V \mid g^{+}(a) \subseteq A_{1}\right\}, \underline{a p r}_{g}^{-}\left(A_{1}\right)=\left\{a \in V \mid g^{-}(a) \subseteq A_{1}\right\}, \\
\overline{a p r}_{g}^{+}\left(A_{1}\right)=\left\{a \in V \mid g^{+}(a) \cap A_{1} \neq \varnothing\right\}, \overline{a p r}_{g}^{-}\left(A_{1}\right)=\left\{a \in V \mid g^{-}(a) \cap A_{1} \neq \varnothing\right\} .
\end{gathered}
$$


Example 6. Consider the Middle East conflict analysis of Table 6. Let $A_{1}=\left\{u_{1}, u_{2}, u_{3}, u_{5}\right\} \subseteq U$. Then, $\underline{a p r}_{g}^{+}\left(A_{1}\right)=\left\{A_{1}, a_{3}, a_{4}\right\}$ and $\underline{\text { apr }_{g}^{-}}\left(A_{1}\right)=\left\{A_{1}, a_{4}\right\}$.

According to Reference [4]:

The agreement subset $=\left\{a_{3}\right\}$, the disagreement subset $=\varnothing$, and

The neutral subset $=\left\{A_{1}, a_{2}, a_{4}, a_{5}\right\}$.

It is noted that for $A_{1}=\left\{u_{1}, u_{2}, u_{3}, u_{5}\right\} \subseteq U$ in the conflict analysis of Table $5, a_{3}$ and $a_{4}$ behave alike, while they have been placed in different alliances. Now, we propose a new and general algorithm for a conflict analysis model which is immaculate from the above flaw.

Definition 9. Let $S=(U, V, \widetilde{g})$ be the Middle East conflict situation and $G: I \rightarrow \mathcal{P}(V \times V)$ be a set valued mapping from $I$ to $\mathcal{P}(V \times V)$, where $I \subseteq U$ denotes the set of parameters or agents and $\mathcal{P}(V \times V)$ the set of all subsets of $V \times V$. If $\varnothing \neq G(u)$ is a preference relation for all $u \in I$, then $(G, I)$ is called a soft preference relation, where:

$$
G(u)=\left\{a_{i} \succeq a_{j} \mid \widetilde{g}\left(a_{i}, u\right) \geq \widetilde{g}\left(a_{j}, u\right), a_{i}, a_{j} \in V\right\}
$$

and:

$$
\widetilde{g}: V \times U \rightarrow\{-, 0,+\},
$$

such that $\widetilde{g}(a, u)=w$, where $w \in\{-, 0,+\}$ and $u \in U, a \in V$. In fact $\widetilde{g}$ is more general than Sun et al.'s $g$ of Reference [4].

Definition 10. If $(G, I)$ is a soft preference relation over $V$, then there is an associated dominance relation, denoted by $\operatorname{Dom}(\mathcal{G}, I)$, which is defined as:

$$
\mathcal{D} o \min \left(\mathcal{G}_{1}, I\right)=\bigcap_{u \in I} G(u) .
$$

For any $a \in V$, define the soft dominance classes by:

$$
[a]_{\mathcal{D} o \min \left(\mathcal{G}_{1}, I\right)}^{+}=\left\{a_{i} \in V: a_{i} \succeq_{\mathcal{D} o \min \left(\mathcal{G}_{1}, I\right)} a\right\}
$$

and:

$$
[a]_{\mathcal{D} o \min \left(\mathcal{G}_{1}, I\right)}^{-}=\left\{a_{i} \in V: a_{i} \preceq_{\mathcal{D} o \min \left(\mathcal{G}_{1}, I\right)} a\right\},
$$

which represent the $(\mathcal{G}, I)$-dominating set and $(\mathcal{G}, I)$-dominated set with respect to $a \in V$ of the $\mathcal{I} \mathcal{S}=(U, V, \widetilde{g})$, respectively. The class $[a]_{\mathcal{D} o \min \left(\mathcal{G}_{1}, I\right)}^{+}$describes the set of all those objects that dominate a and $[a]_{\mathcal{D} o \min \left(\mathcal{G}_{1}, I\right)}^{-}$ describes the set of all those objects that are dominated by $a$, in terms of $\mathcal{D} o$ min $\left(\mathcal{G}_{1}, I\right)$, where:

$$
\mathcal{D} o \min \left(\mathcal{G}_{1}, I\right)^{+}=\left\{a \in V:[a]_{\mathcal{D} o \min \left(\mathcal{G}_{1}, I\right)}^{+}\right\}
$$

and:

$$
\mathcal{D} o \min \left(\mathcal{G}_{1}, I\right)^{-}=\left\{a \in V:[a]_{\mathcal{D} o \min \left(\mathcal{G}_{1}, I\right)}^{-}\right\} .
$$

For $A_{2} \subseteq V$ the lower and upper approximations with respect to the $\left(\mathcal{G}_{1}, I\right)$-dominating set and $\left(\mathcal{G}_{1}, I\right)$-dominated set are:

$$
\begin{gathered}
\left(\underline{A_{2}}\right)_{\mathcal{D} o \min \left(\mathcal{G}_{1}, I\right)^{+}}=\left\{a \in V:[u]_{\mathcal{D} o \min \left(\mathcal{G}_{1}, I\right)}^{+} \subseteq A_{2}\right\} \\
\left(\overline{A_{2}}\right)_{\mathcal{D}_{\min \left(\mathcal{G}_{1}, I\right)^{+}}}=\left\{a \in V:[u]_{\mathcal{D} o \min \left(\mathcal{G}_{1}, I\right)}^{-} \cap A_{2}: \neq \varnothing\right\} .
\end{gathered}
$$

Similarly:

$$
{\underline{\left(A_{2}\right.}}_{\mathcal{D}_{o \min \left(\mathcal{G}_{1}, I\right)^{-}}}=\left\{a \in V:[u]_{\mathcal{D} o \min \left(\mathcal{G}_{1}, I\right)}^{-} \subseteq A_{2}\right\}
$$




$$
\left(\overline{A_{2}}\right)_{\mathcal{D} o \min \left(\mathcal{G}_{1}, I\right)^{-}}=\left\{a \in V:[u]_{\mathcal{D} o \min \left(\mathcal{G}_{1}, I\right)}^{+} \cap A_{2}: \neq \varnothing\right\} .
$$

Now, the agreement subset $=\left(\underline{A_{2}}\right)_{\mathcal{D} o \min \left(\mathcal{G}_{1}, I\right)^{+}}-\left(\underline{A_{2}}\right)_{\mathcal{D} \text { o } \min \left(\mathcal{G}_{1}, I\right)^{-}}$,

The disagreement subset $=\left(\underline{A_{2}}\right)_{\mathcal{D} o \min \left(\mathcal{G}_{1}, I\right)^{-}}-\left(\underline{A_{2}}\right)_{\mathcal{D} o \min \left(\mathcal{G}_{1}, I\right)^{+}}$, and

The neutral subset $=V-($ Agreement subset $\cup$ Disagreement subset $)$.

Example 7. Consider the Middle East conflict analysis of Table 6. Let $I=\left\{u_{1}, u_{2}, u_{3}, u_{5}\right\} \subseteq U$. Then by our second proposed technique:

The agreement subset $=\varnothing$, the disagreement subset $=\left\{a_{3}, a_{4}\right\}$, and

The neutral subset $=\left\{A_{1}, a_{2}, a_{5}\right\}$.

Thus for $I=\left\{u_{1}, u_{2}, u_{3}, u_{5}\right\} \subseteq U$ in the conflict situation, the agents in I do not agree on a feasible strategy in universe $V$. The example of the Middle East conflict analysis does not necessarily reflect the present day situation in this region, but is used here only as an illustration of the basic ideas considered in this paper.

Aims and Advantages/Applications of the Proposed Model

(1) The proposed model rightly classifies the agents.

(2) The proposed model answers questions (i) and (ii) of Deja in a better way than Sun et al.

(3) Another advantage of the proposed model is that, it can be used for ranking the feasible alternatives simpler than other existing techniques/models.

(4) The proposed model can be used for reduction of attributes.

(5) Using the proposed model, one can find two types of rough degree, precision and approximate quality, and their relationship.

(6) The proposed model can be applied to multi-criteria and multi-decision conflict analysis problems.

(7) As a real world world application, the proposed model can be applied to solve the problem of determining the governor election results in Indonesia.

\section{Conclusions and Future Work}

This paper aimed to mainly discuss the Middle East conflict. The proposed model was studied initially by Pawlak in Reference [3] with the help of rough set theory on a single universe. Subsequently, drawbacks were highlighted by Deja, where he put some questions related to conflict analysis. Sun et al. developed a new conflict analysis model and tried to answer the Deja's questions with help of rough set theory over two universes. But their model carried some shortcomings that made the study of conflict analysis more ambiguous. In this paper, a new conflict analysis model is proposed to study the Middle East conflict analysis problem with the help of the soft preference relation and soft dominance relation. Further, attempted answers to Deja's questions were made in a positive manner. The proposed model can be extended to study other conflicts around the world. The present conflict analysis model depicts the real spirit of the conflict situation. Beginning with some basic properties of soft dominance classes, we discussed Pawlak's properties in the framework of the proposed new rough sets. A connection between the proposed new model and topology was established. Two types of approximate precision, rough degree, and their relationship were also discussed. Further, two types of approximate quality and the relationships between approximate quality and rough degree were also a part of the current paper. The same relation is established between approximate precision and approximate quality. Last, we answered the questions posed by Deja. We believe that this work would offer foundations for further study of the theory of conflict analysis.

In our future study of conflict analysis, the following topics might be considered:

(1) To apply the proposed model to multigranulation decision theoretic rough sets.

(2) To apply this model to labor-management negotiation conflict analysis.

(3) To apply this model to game theory. 
Author Contributions: All the authors of this paper contributed equally. They have read and approved the final version of the paper.

Funding: Sun Young Jang was supported by Basic Science Research Program through the National Research Foundation of Korea by the Ministry of Education, Science and Technology (NRF-2018-07042748) and Choonkil Park was supported by Basic Research Program through the National Research Foundation of Korea funded by the Ministry of Education, Science and Technology (NRF-2017R1D1A1B04032937).

Acknowledgments: We would like to express our sincere thanks to the anonymous referees of this paper for their interest in our work, and also for spending their valuable time in reading this manuscript carefully and giving their useful comments for improving the earlier version of the paper.

Conflicts of Interest: The authors declare no conflict of interest.

\section{References}

1. Nguyen, N.T. Consensus system for solving conflicts in distributed system. Inf. Sci. 2002, 147, 91-122. [CrossRef]

2. Deja, R. Conflict analysis. Int. J. Intell. Syst. 2002, 17, 235-253. [CrossRef]

3. Pawlak, Z. An inquiry into anatomy of conflicts. J. Inf. Sci. 1998, 109, 65-68. [CrossRef]

4. Sun, B.; Ma, W.; Zhao, H. Rough set based conflict analysis model and method over two universes. Inf. Sci. 2016, 372, 111-125. [CrossRef]

5. Molodtsov, D. Soft set theory-First results. Comput. Math. Appl. 1999, 37, 19-31. [CrossRef]

6. Maji, P.K.; Biswas, R.; Roy, A.R. Soft set theory. Comput. Math. Appl. 2003, 45, 555-562. [CrossRef]

7. Ali, M.I.; Feng, F.; Liu, X.Y.; Min, W.K.; Shabir, M. On some new operations in soft set theory. Comput. Math. Appl. 2009, 57, 1547-1553. [CrossRef]

8. Ali, M.I. Another view on reduction of parameters in soft sets. Appl. Soft Comput. 2012, 12, $1814-1821$. [CrossRef]

9. Alcantud, J.C.R.; de Andres Calle, R.; Torrecillas, M.J.M. Hesitant fuzzy worth: An innovative ranking methodology for hesitant fuzzy subsets. Appl. Soft Comput. 2016, 38, 232-243. [CrossRef]

10. Alcantud, J.C.R.; Torra, V. Decomposition theorems and extension principles for hesitant fuzzy sets. Inf. Fusion 2018, 41, 48-56. [CrossRef]

11. Cagman, N.; Enginoglu, S. Soft matrix theory and its decision making. Math. Appl. 2010, 59, 3308-3314.

12. Cagman, N.; Enginoglu, S. Soft set theory and uni-int decision-making. Eur. J. Oper. Res. 2010, $207,848-855$. [CrossRef]

13. Jiang, Y.; Liu, H.; Tanga, Y.; Chen, Q. Semantic decision-making using ontology based soft sets. Math. Comput. Model. 2011, 53, 1140-1149. [CrossRef]

14. Ma, X.; Zhan, J.; Ali, M.I.; Mehmood, N. A survey of decision making methods based on two classes of hybrid soft set models. Artif. Intell. Rev. 2018, 49, 511-529. [CrossRef]

15. Ma, X.; Liu, Q.; Zhan, J. A survey of decision making methods based on certain hybrid soft set models. Artif. Intell. Rev. 2017, 47, 507-530. [CrossRef]

16. Roy, A.R.; Maji, P.K. A fuzzy soft set theoretic approach to decision making problems. J. Comput. Appl. Math. 2007, 203, 412-418. [CrossRef]

17. Slowinski, R.; Greco, S.; Matarazzo, B. Rough-Set-Based Decision support. In Search Methodologies; Springer: Boston, MA, USA, 2014; pp. 557-607.

18. Torra, V. Hesitant fuzzy sets. Int. J. Intell. Syst. 2010, 25, 529-539. [CrossRef]

19. Zhan, J.; Zhu, K. Reviews on decision making methods on (fuzzy) soft sets and rough soft sets. J. Intell. Fuzzy Syst. 2015, 29, 1169-1176. [CrossRef]

20. Zhan, J.; Yu, B.; Fotea, V.E. Characterizations of two kinds of hemiring based on probability spaces. Soft Comput. 2016, 20, 637-648. [CrossRef]

21. Zhan, J.; Liu, Q.; Zhu, W. Another approach to rough soft hemirings and corresponding decision making. Soft Comput. 2017, 21, 3769-3780. [CrossRef]

22. Zhan, J.; Liu, Q.; Herawan, T. A novel soft rough set: Soft rough hemirings and its multicriteria group decion making. Appl. Soft Comput. 2017, 54, 393-402. [CrossRef]

23. Zhan, J.; Zhu, K. A novel soft rough fuzzy set: Z-soft rough fuzzy ideals of hemirings and corresponding decision making. Soft Comput. 2017, 21, 1923-1936. [CrossRef] 
24. Zhan, J.; Ali, M.I.; Mehmood, N. On a novel uncertain soft set model: Z-soft fuzzy rough set model and corresponding decision making methods. Appl. Soft Comput. 2017, 56, 446-457. [CrossRef]

25. Braszczynski, J.; Greco, S.; Slowinski, R. Multi-criteria classification a new scheme for application of dominance-based decision rules. Eur. J. Oper. Res. 2007, 181, 1030-1044. [CrossRef]

26. Dubois, D.; Fargier, H.; Prade, H. Ordinal and probabilistic representations of acceptance. J. Artif. Intell. Res. 2004, 22, 23-56. [CrossRef]

27. Orlowska, M.; Orlowski, M. Maintenance of knowledge in dynamic information systems. In Intelligent Decision Support, Handbook of Applications and Advances of the Rough Set Theory; Slowinski, R., Ed.; Kluwer Academic Publishers: Dordrecht, The Netherlands, 1992; pp. 315-330.

28. Hu, Y.C.; Chiu, Y.J. Incorporating Grey Total Influence into Tolerance Rough Sets for Classification Problems. Appl. Sci. 2018, 8, 1173. [CrossRef]

29. Azam, N.; Yao, J.T. Analyzing Uncertainties of Probabilistic Rough Set Regions with Game-theoretic Rough Sets. Int. J. Approx. Reason. 2014, 55, 142-155. [CrossRef]

30. Zhang, Y. Optimizing Gini Coefficient of Probabilistic Rough Set Regions using Game-Theoretic Rough Sets. In Proceedings of the 26th Annual IEEE Canadian Conference on Electrical and Computer Engineering (CCECE'13), Regina, SK, Canada, 5-8 May 2013; Volume 5-8, pp. 699-702.

31. Yao, Y.Y. The two sides of the theory of rough sets. Knowl.-Based Syst. 2015, 80, 67-77. [CrossRef]

32. Arena, P.; Fazzino, S.; Fortuna, L.; Maniscalco, P. Game theory and non-linear dynamics: The Parrondo Paradox case study. Chaos Solitons Fractals 2003, 17, 545-555. [CrossRef]

(c) 2018 by the authors. Licensee MDPI, Basel, Switzerland. This article is an open access article distributed under the terms and conditions of the Creative Commons Attribution (CC BY) license (http://creativecommons.org/licenses/by/4.0/). 